\title{
Structure of a new multicomponent stannide and comments on the Yarmolyuk-Kripyakevich criterion for Frank-Kasper phases
}

\author{
Volodymyr GVOZDETSKYI ${ }^{1,2} *$, Roman GLADYSHEVSKII ${ }^{1}$, Viktor HLUKHYY ${ }^{2}$ \\ ${ }^{1}$ Department of Inorganic Chemistry, Ivan Franko National University of Lviv, \\ Kyryla i Mefodiya St. 6, 79005 Lviv, Ukraine \\ ${ }^{2}$ Department of Inorganic Chemistry, Technical University of Munich, \\ Lichtenbergstr. 4, 85747 Garching, Germany \\ * Corresponding author. Tel: +380 32 2394506; e-mail: volodymyr.gvozdetskyi@ gmail.com
}

Received September 26, 2014; accepted December 24, 2014; available on-line September 1, 2015

\begin{abstract}
The structure of a new stannide, $\mathrm{Nb}_{4.4} \mathrm{~V}_{1.6} \mathrm{Ni}_{6} \mathrm{Sn}_{0.9}$, was refined from $\mathrm{X}$-ray single-crystal diffraction data $\left(P 6_{3} / m m c, a=4.9433(5), c=17.935(3) \AA\right)$. It appeared to be formed by two substructures: $\mathrm{Nb}_{5} \mathrm{Ni}_{6.84} \mathrm{~V}_{0.16} \mathrm{Sn}_{0.84}$ and $\mathrm{V}_{12} \mathrm{Sn}$, in the approximate ratio 9:1. The first substructure, $\mathrm{Nb}_{5} \mathrm{Ni}_{6}\left(\mathrm{Ni}_{0.84} \mathrm{~V}_{0.16}\right) \mathrm{Sn}_{0.84}$, is an ordering variant of the $\mathrm{Cs}_{6} \mathrm{~K}_{7}$ type and belongs to the family of tetrahedrally close-packed structures, in which the atoms are surrounded by $P$ - (16 vertices), $Q$ - (15 vertices), $R$ - (14 vertices), and $X$ - (12 vertices) Frank-Kasper polyhedra. The second substructure, $\mathrm{V}_{12} \mathrm{Sn}$, is built from Q-polyhedra and 20-vertex pseudo-Frank-Kasper polyhedra and reveals similarities with the $\mathrm{Be}_{12} \mathrm{Ti}$ type. The list of known structures with Frank-Kasper polyhedra was updated and new features governing this family of compounds were discovered. It was found that, among the number of possible hypothetical structures, only one combination derived from the Yarmolyuk-Kripyakevich criterion, is observed for a particular number of icosahedra.
\end{abstract}

Intermetallics / Tetrahedrally close-packed structure / Frank-Kasper polyhedra / Yarmolyuk-Kripyakevich criterion

\section{Introduction}

Phases with structures that are built from FrankKasper polyhedra represent a broad class: 2036 phases (polytypes of Friauf-Laves phases not included) are compiled in [1]. The crystal chemical features of these compounds have been thoroughly described and interpreted (see for example [2-6]). An important observation is the linear dependence between the weight-averaged coordination number and the icosahedron content, known as the "YarmolyukKripyakevich criterion" [4]. From a genealogical point of view, the structure belonging to this family take their origin in the simple structure types $\mathrm{Cr}_{3} \mathrm{Si}$ (Pearson symbol $c P 8$, space group $P m-3 n$ ), $\mathrm{MgZn}_{2}$ (hP12, $\left.P 6_{3} / m m c\right)$, and $\mathrm{Zr}_{4} \mathrm{Al}_{3}$ (hP7, P6/mmm).

All the atoms in these structures are surrounded by Frank-Kasper polyhedra, of which there exist four kinds: P- (16 vertices), Q- (15 vertices), R(14 vertices), and $\mathrm{X}$ - (12 vertices) polyhedra. The Frank-Kasper polyhedra have only triangular faces and surface coordination numbers 5 (for the 12-vertex icosahedron), or 5 and 6 . The space is filled by interpenetrating centered Frank-Kasper polyhedra in a way so that each atom at the vertex of a polyhedron is itself the central atom of another polyhedron. The interstices are exclusively tetrahedral (the tetrahedra are moderately distorted, with ratios between the longest and the shortest edges of at most $\sim 4 / 3$ ), which is at the origin of the term "tetrahedrally closepacked structures" (t.c.p.). The structural formula of any of the structures can be presented as $\left(\mathrm{R}_{3} \mathrm{X}\right)_{i}\left(\mathrm{PX}_{2}\right)_{j}\left(\mathrm{Q}_{2} \mathrm{R}_{2} \mathrm{X}_{3}\right)_{k}$, i.e. as a combination of the $\mathrm{Cr}_{3} \mathrm{Si}\left(\mathrm{R}_{3} \mathrm{X}\right), \mathrm{MgZn}_{2}\left(\mathrm{PX}_{2}\right)$, and $\mathrm{Zr}_{4} \mathrm{Al}_{3}\left(\mathrm{Q}_{2} \mathrm{R}_{2} \mathrm{X}_{3}\right)$ prototypes, mentioned above.

No compounds were so far known in the quaternary system $\mathrm{Nb}-\mathrm{V}-\mathrm{Ni}-\mathrm{Sn}$ and only one compound, $\mathrm{NbNi}_{2} \mathrm{Sn}$, has been reported in the ternary system $\mathrm{Nb}-\mathrm{Ni}-\mathrm{Sn}$ [7]. Its structure belongs to the $\mathrm{MnCu}_{2} \mathrm{Al}$ type $(c F 12, F m-3 m)$, like the structure of the compound $\mathrm{VNi}_{2} \mathrm{Sn}$ [8], reported in the ternary system $\mathrm{V}-\mathrm{Ni}-\mathrm{Sn}$. In the latter system we also observed the existence of a compound with approximate composition $\mathrm{VNiSn}$ and $\mathrm{Ni}_{2} \mathrm{In}$-type structure $\left(h P 6, \mathrm{PG}_{3} / \mathrm{mmc}\right)$ [9]. In the ternary system $\mathrm{Nb}-\mathrm{V}-\mathrm{Ni}$ the following compounds form: $\mathrm{NbVNi}$, $\mathrm{Nb}_{5.3} \mathrm{~V}_{3.7} \mathrm{Ni}_{4}$, and $\mathrm{Nb}_{0.5} \mathrm{~V}_{0.5} \mathrm{Ni}_{3}$ [10]. The structures of the two first compounds belong to the types $\mathrm{MgZn}_{2}$ $\left(h P 12, \quad P 6_{3} / m m c\right)$ and $\quad \mathrm{W}_{6} \mathrm{Fe}_{7} \quad(h R 39, \quad R-3 m)$, respectively, which are members of the family of 
tetrahedrally close-packed structures. The $\mathrm{Nb}_{0.5} \mathrm{~V}_{0.5} \mathrm{Ni}_{3}$ compound has $\mathrm{TiCu}_{3}$-type structure (oP8, Pmmn).

The aim of this work was to determine the structure of a new stannide found in the quaternary system $\mathrm{Nb}-\mathrm{V}-\mathrm{Ni}-\mathrm{Sn}$. The discovery of a t.c.p substructure motivated us to update the list of known structure types with Frank-Kasper polyhedra and to have a closer look at the structural features of this family and the validity of the YarmolyukKripyakevich criterion.

\section{Experiment and results}

Starting materials for the intended synthesis of ternary $\mathrm{V}-\mathrm{Ni}-\mathrm{Sn}$ alloys were ingots of vanadium, nickel, and tin, with purities better than $99.85 \%$. Samples with a mass of $\sim 1 \mathrm{~g}$ were synthesized in an arc furnace with a copper water-cooled hearth, using a tungsten electrode under argon atmosphere. A sample of equiatomic composition VNiSn was sealed in a niobium container and placed into the water-cooled chamber of an induction furnace (Hüttinger Elektronik, Freiburg, TIG 2.5/300). It was first heated under flowing argon up to $1300 \mathrm{~K}$ and held at this temperature for $0.5 \mathrm{~h}$ (the temperature was controlled through a Sensor Therm Metis MS06 pyrometer with an accuracy of $\pm 5 \mathrm{~K})$. Then the sample was slowly cooled to $1170 \mathrm{~K}$ and held at that temperature for $1 \mathrm{~h}$. After that it was cooled by regulating the frequency with a resulting average cooling rate of $10 \mathrm{~K} / \mathrm{min}$, maintained until the furnace was switched off. It became clear that a reaction had taken place between the ternary sample and the niobium container. Single crystals were selected for further examinations. The EDX analyses (performed by means of a JEOL 5900LV scanning electron microscope) of the single crystals revealed the following composition (in at.\%): $\mathrm{Nb}$ 33(5), V 17(3), Ni 46(7), Sn 4(2).

Single-crystal diffraction data were collected at room temperature on an Oxford Xcalibur $3 \mathrm{CCD}$ diffractometer (graphite monochromator, MoKa radiation, $\lambda=0.71073 \AA$ ). An empirical absorption correction was applied to the data [11]. Relevant experimental details and crystallographic data for the $\mathrm{Nb}_{4.4} \mathrm{~V}_{1.6} \mathrm{Ni}_{6} \mathrm{Sn}_{0.9}$ are listed in Table 1 .

Analysis of the diffraction data revealed hexagonal Laue symmetry. The systematic absences and the $E$-value statistics indicated the centrosymmetric space group $P 6_{3} / m m c$, which was used for the structure solution. Starting atomic coordinates were deduced from an interpretation of direct methods (program SHELXS-97 [12]); the atoms were distributed among six atom sites occupying the following Wyckoff positions: $\mathrm{Nb}$ atoms in two positions $4 f$ and one $2 b, \mathrm{Ni}$ atoms in $12 k$ and $2 a, \mathrm{Sn}$ atoms in $2 c$ (Table 2). The refinement revealed significant electron density residuals on the difference Fourier map, which were taken into consideration by "splitting" three sites $(4 f$, $2 b$, and $12 k$ ). As a consequence three additional positions, partly occupied by $\mathrm{V}$ atoms, were introduced $(4 e, 2 d$, and $12 k)$. The refinement was further improved by considering a statistical mixture $\mathrm{Nb} / \mathrm{V}$ on the remaining site in position $4 f$ and a mixture $\mathrm{Ni} / \mathrm{V}$ in $2 a$, whereas the position $2 c$ was found to be partly occupied by $\mathrm{Sn}$ atoms. The atom ratio $\mathrm{Nb} / \mathrm{V}$ of the sites in position $4 f$ and in the splitpositions $4 f / 4 e, 2 b / 2 d$, and the atom ratio $\mathrm{Ni} / \mathrm{V}$ in the split-position $12 k / 12 k$ refined to similar values $(\sim 0.9 / 0.1)$, and were constrained to identical values in the last cycles of the refinement. The element ratio of the statistical mixture $\mathrm{Ni} / \mathrm{V}$ in position $2 a$ was different $\quad(\sim 0.7 / 0.3)$, therefore it was refined separately. The final refinement of the structure was performed with anisotropic displacement parameters for all the sites (constraining the values of the split sites).

The structure of $\mathrm{Nb}_{4.4} \mathrm{~V}_{1.6} \mathrm{Ni}_{6} \mathrm{Sn}_{0.9}$ can be described in terms of two substructures: $\mathrm{Nb}_{5} \mathrm{Ni}_{6.84} \mathrm{~V}_{0.16} \mathrm{Sn}_{0.84}$ $(88 \%)$ and $\mathrm{V}_{12} \mathrm{Sn}(12 \%)$ (Tables 3 and 4$)$. The ideal formula of the former substructure is $\mathrm{Nb}_{5} \mathrm{Ni}_{7} \mathrm{Sn}$. Partial substitution of "five-valent" $\mathrm{V}$ for $\mathrm{Ni}$ (represented in the site Ni5/V5) is compensated by a decrease of the content of "four-valent" $\mathrm{Sn}$ (vacancies on site Sn6), according to the apparent formula $\mathrm{Nb}_{5} \mathrm{Ni}_{7-x} \mathrm{~V}_{x} \mathrm{Sn}_{1-x} \quad(x=0.16)$. Both substructures are described by Pearson symbol $h P 26$ and space group $P 6_{3} / m m c$. The structure of $\mathrm{Nb}_{5} \mathrm{Ni}_{7} \mathrm{Sn}$ $\left(\mathrm{Nb}_{5} \mathrm{Ni}_{6.84} \mathrm{~V}_{0.16} \mathrm{Sn}_{0.84}\right)$ is a ternary ordering variant of the type $\mathrm{Cs}_{6} \mathrm{~K}_{7}$ [13] and the structure of $\mathrm{V}_{12} \mathrm{Sn}$ is a deformation variant of the type $\mathrm{TiBe}_{12}(h P 13$, $P 6 / \mathrm{mmm}$ ) [14] with two-fold cell volume.

Interatomic distances within the two substructures in Nb4.4V1.6Ni6Sn0.9 are presented in Table 5. Considered independently, both substructures present distances in good agreement with expected values. The $\mathrm{Nb}$ atoms in the substructure Nb5Ni6.84V0.16Sn0.84 are surrounded by 14-, 15-, and 16-vertex Frank-Kasper coordination polyhedra, the $\mathrm{Sn}$ atoms are located inside 15-vertex FrankKasper polyhedra, and the $\mathrm{Ni}$ and $\mathrm{Ni} / \mathrm{V}$ atoms at the centers of 12-vertex polyhedra (icosahedra). In the V12Sn substructure the $\mathrm{V}$ atoms are surrounded by 12-, 14-, and 15-vertex Frank-Kasper polyhedra, and by 20 -vertex polyhedra; the $\mathrm{Sn}$ atoms are inside 15-vertex Frank-Kasper polyhedra.

\section{Discussion}

The four kinds of Frank-Kasper polyhedron, P- (16 vertices), Q- (15 vertices), R- (14 vertices), and $\mathrm{X}$ - (12 vertices, icosahedron), which appear in the members of the family of tetrahedrally close-packed structures, are shown in Fig. 1. 38 structure types built from Frank-Kasper polyhedra (as mentioned above, polytypes of the Friauf-Laves phases, represented by only PX2 fragments, are not included) and their structural formulas (R3X)i(PX2)j(Q2R2X3)k are listed in Table 6. 
Table 1 Experimental details and crystallographic data for $\mathrm{Nb}_{4.4} \mathrm{~V}_{1.6} \mathrm{Ni}_{6} \mathrm{Sn}_{0.9}$.

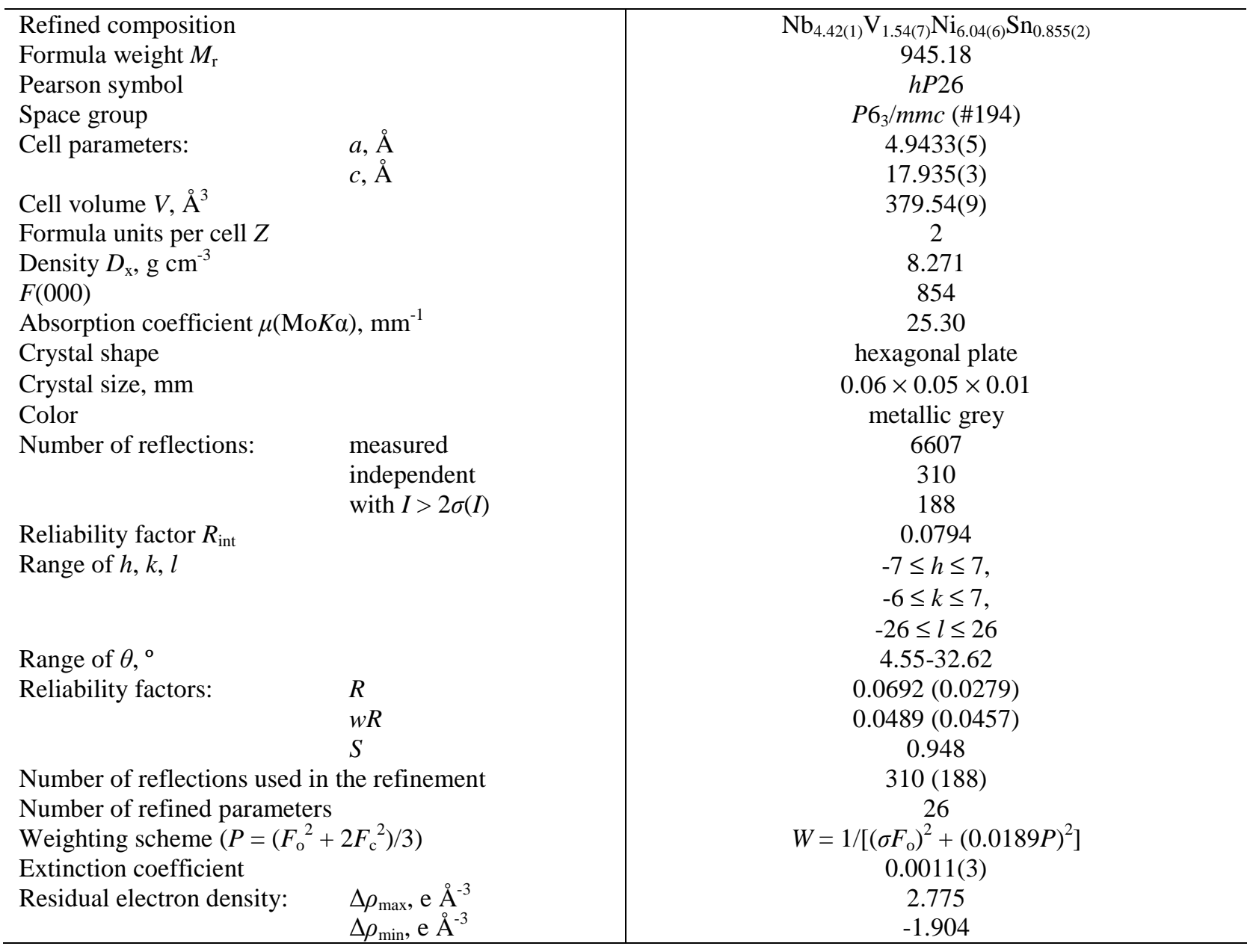

The general formula $\left(\mathrm{R}_{3} \mathrm{X}\right)_{i}\left(\mathrm{PX}_{2}\right)_{j}\left(\mathrm{Q}_{2} \mathrm{R}_{2} \mathrm{X}_{3}\right)_{k}$ can be rewritten as $\mathrm{P}_{j} \mathrm{Q}_{2 k} \mathrm{R}_{3 i+2 k} \mathrm{X}_{i+2 j+3 k}$, which allows better understanding the linear dependence between the average coordination number and the icosahedron content.

Let us consider the general case of a tetrahedrally close-packed structure with $N$ atoms in the unit cell. The atoms are distributed over different atom sites, where $m_{1}$ is the multiplicity of the first atom site, $m_{2}$ of the second atom site, $\ldots$ and $m_{n}$ of the $n^{\text {th }}$ atom site:

$m_{1}+m_{2}+\ldots+m_{n}=N$.

Considering the different atomic environments of the sites:

$m_{\mathrm{P} 1}+m_{\mathrm{P} 2}+\ldots+m_{\mathrm{P} n}=N(\mathrm{P})$,

$m_{\mathrm{Q} 1}+m_{\mathrm{Q} 2}+\ldots+m_{\mathrm{Q} n}=N(\mathrm{Q})$

$m_{\mathrm{R} 1}+m_{\mathrm{R} 2}+\ldots+m_{\mathrm{R} n}=N(\mathrm{R})$,

$m_{\mathrm{X} 1}+m_{\mathrm{X} 2}+\ldots+m_{\mathrm{X} n}=N(\mathrm{X})$

where $m_{\mathrm{P} n}, m_{\mathrm{Q} n}, m_{\mathrm{R} n}$, and $m_{\mathrm{X} n}$ are the multiplicities of the atom sites, which are at the centers of $\mathrm{P}_{-}, \mathrm{Q}_{-}, \mathrm{R}-$, and X-polyhedra, respectively; and $N(\mathrm{P}), N(\mathrm{Q}), N(\mathrm{R})$, and $N(\mathrm{X})$ are the total numbers of atoms centering $\mathrm{P}-$, Q-, R-, and X-polyhedra in the unit cell.
Obviously:

$N(\mathrm{P})+N(\mathrm{Q})+N(\mathrm{R})+N(\mathrm{X})=N$.

These numbers are related to the numbers used above by the number of formula units per cell, $Z$ :

$N_{16}+N_{15}+N_{14}+N_{12}=N / Z$,

where $Z$ is the largest common divisor, and $N_{16}, N_{15}$, $N_{14}$, and $N_{12}$ are the number of corresponding polyhedra.

Each t.c.p. structure is characterized by a certain number of $\mathrm{X}$ - (12 vertices) polyhedra, i.e. the case when P- (16 vertices) and/or Q- (15 vertices), R- (14 vertices) polyhedra are present without X-polyhedra does not exist. The values $N_{16}, N_{15}, N_{14}$, and $N_{12}$ are not independent and $N_{12}=\mathrm{f}\left(N_{16}, N_{15}, N_{14}\right)$. This dependence can be determined from the correlation between the coefficients $i, j$, and $k$ in $\left(\mathrm{R}_{3} \mathrm{X}\right)_{i}\left(\mathrm{PX}_{2}\right)_{j}\left(\mathrm{Q}_{2} \mathrm{R}_{2} \mathrm{X}_{3}\right)_{k}=\mathrm{P}_{j} \mathrm{Q}_{2 k} \mathrm{R}_{3 i+2 k} \mathrm{X}_{i+2 j+3 k}$. $N_{12}=\mathrm{p} \cdot N_{16}+\mathrm{q} \cdot N_{15}+\mathrm{r} \cdot N_{14}$,

where $\mathrm{p}$ is the number of $\mathrm{N}_{12}$ polyhedra "provided" by one 16-vertex P-polyhedron, etc.

$i+2 j+3 k=\mathrm{p} \cdot j+\mathrm{q} \cdot 2 k+\mathrm{r} \cdot(3 i+2 k)$.

As can be seen from Eq. $9,2 j=\mathrm{p} \cdot j$ and $i=\mathrm{r} \cdot 3 i$. Thus $\mathrm{p}=2, \mathrm{r}=1 / 3$, and $\mathrm{q}=7 / 6$. 
Table 2 Atomic coordinates, displacement parameters $\left(\AA^{2}\right)$ and site occupancies for $\mathrm{Nb}_{4.4} \mathrm{~V}_{1.6} \mathrm{Ni}_{6} \mathrm{Sn}_{0.9}(h P 26$, $\left.P 6_{3} / m m c, a=4.9433(5), c=17.935(3) \AA, R=0.0279\right)$.

\begin{tabular}{|c|c|c|c|c|c|c|}
\hline Site & $\begin{array}{l}\text { Wyckoff } \\
\text { position }\end{array}$ & $x$ & $y$ & $z$ & $U_{\text {eq }}$ & $\begin{array}{c}\text { Site } \\
\text { occupancy }\end{array}$ \\
\hline $\begin{array}{c}\mathrm{Nb1} \\
\mathrm{V} 1\end{array}$ & $\begin{array}{l}4 f \\
4 f\end{array}$ & $1 / 3$ & $2 / 3$ & $0.52253(6)$ & $0.0119(3)$ & $\begin{array}{l}0.884(2) \\
0.116(2)\end{array}$ \\
\hline $\begin{array}{c}\mathrm{Nb} 2 \\
\mathrm{~V} 2\end{array}$ & $\begin{array}{l}4 f \\
4 e\end{array}$ & $\begin{array}{l}1 / 3 \\
0\end{array}$ & $\begin{array}{l}2 / 3 \\
0\end{array}$ & $\begin{array}{l}0.67866(7) \\
0.1781(11)\end{array}$ & $\begin{array}{l}0.0122(3) \\
0.0122(3)\end{array}$ & $\begin{array}{l}0.884(2) \\
0.116(2)\end{array}$ \\
\hline $\begin{array}{l}\mathrm{Nb3} \\
\mathrm{V} 3\end{array}$ & $\begin{array}{l}2 b \\
2 d\end{array}$ & $\begin{array}{c}0 \\
1 / 3\end{array}$ & $\begin{array}{c}0 \\
2 / 3\end{array}$ & $\begin{array}{l}1 / 4 \\
3 / 4\end{array}$ & $\begin{array}{l}0.0088(4) \\
0.0088(4)\end{array}$ & $\begin{array}{l}0.884(2) \\
0.116(2)\end{array}$ \\
\hline $\begin{array}{l}\mathrm{Ni4} \\
\mathrm{V} 4\end{array}$ & $\begin{array}{l}12 k \\
12 k\end{array}$ & $\begin{array}{l}0.1673(2) \\
0.505(2)\end{array}$ & $\begin{array}{l}0.3346(4) \\
0.010(4)\end{array}$ & $\begin{array}{l}0.11353(5) \\
0.6157(6)\end{array}$ & $\begin{array}{l}0.0097(2) \\
0.0097(2)\end{array}$ & $\begin{array}{l}0.884(2) \\
0.116(2)\end{array}$ \\
\hline $\begin{array}{l}\text { Ni5 } \\
\text { V5 }\end{array}$ & $\begin{array}{l}2 a \\
2 a\end{array}$ & 0 & 0 & 0 & $0.0181(10)$ & $\begin{array}{l}0.74(5) \\
0.26(5)\end{array}$ \\
\hline Sn6 & $2 c$ & $1 / 3$ & $2 / 3$ & $1 / 4$ & $0.0106(3)$ & $0.855(2)$ \\
\hline Site & $U_{11}$ & $U_{22}$ & $U_{33}$ & $U_{12}$ & $U_{13}$ & $U_{23}$ \\
\hline $\begin{array}{c}\mathrm{Nb} 1 \\
\mathrm{~V} 1\end{array}$ & $0.0085(4)$ & $0.0085(4)$ & $0.0187(6)$ & $0.0042(2)$ & 0 & 0 \\
\hline $\begin{array}{c}\mathrm{Nb} 2 \\
\mathrm{~V} 2\end{array}$ & $0.0125(4)$ & $0.0125(4)$ & $0.0115(6)$ & $0.0063(2)$ & 0 & 0 \\
\hline $\begin{array}{c}\text { Nb3 } \\
\text { V3 }\end{array}$ & $0.0098(6)$ & $0.0098(6)$ & $0.0069(8)$ & $0.0049(3)$ & 0 & 0 \\
\hline $\begin{array}{c}\mathrm{Ni4} \\
\mathrm{V} 4 \\
\end{array}$ & $0.0092(3)$ & $0.0081(5)$ & $0.0116(4)$ & $0.0040(2)$ & $0.0000(3)$ & $0.0000(5)$ \\
\hline $\begin{array}{l}\mathrm{Ni5} \\
\mathrm{V} 5 \\
\end{array}$ & $0.0079(11)$ & $0.0079(11)$ & $0.038(2)$ & $0.0039(5)$ & 0 & 0 \\
\hline Sn6 & $0.0089(5)$ & $0.00829(5)$ & $0.0139(6)$ & $0.0044(2)$ & 0 & 0 \\
\hline
\end{tabular}

Table 3 Atomic coordinates, displacement parameters $\left(\AA^{2}\right)$ and site occupancies for the substructure $\mathrm{Nb}_{5} \mathrm{Ni}_{6.84} \mathrm{~V}_{0.16} \mathrm{Sn}_{0.84}\left(h P 26, P 6_{3} / m m c, a=4.9433(5), c=17.935(3) \AA\right)$.

\begin{tabular}{|c|c|c|c|c|c|c|c|}
\hline Site & $\begin{array}{l}\text { Wyckoff } \\
\text { position }\end{array}$ & $\begin{array}{c}\text { Polyhedron } \\
\text { type }\end{array}$ & $x$ & $y$ & $z$ & $U_{\text {eq }}$ & $\begin{array}{c}\text { Site } \\
\text { occupancy }\end{array}$ \\
\hline $\mathrm{Nb1}$ & $4 f$ & $\mathrm{P}$ & $1 / 3$ & $2 / 3$ & $0.52253(6)$ & $0.0119(3)$ & 1 \\
\hline $\mathrm{Nb} 2$ & $4 f$ & $\mathrm{R}$ & $1 / 3$ & $2 / 3$ & $0.67866(7)$ & $0.0122(3)$ & 1 \\
\hline $\mathrm{Nb3}$ & $2 b$ & $\mathrm{Q}$ & 0 & 0 & $1 / 4$ & $0.0088(4)$ & 1 \\
\hline $\mathrm{Ni} 4$ & $12 k$ & $X$ & $0.1673(2)$ & $0.3346(4)$ & $0.11353(5)$ & $0.0097(2)$ & 1 \\
\hline $\begin{array}{l}\mathrm{Ni5} \\
\mathrm{V} 5\end{array}$ & $\begin{array}{l}2 a \\
2 a\end{array}$ & $\begin{array}{l}X \\
X\end{array}$ & 0 & 0 & 0 & $0.0181(10)$ & $\begin{array}{l}0.84(5) \\
0.16(5)\end{array}$ \\
\hline Sn6 & $2 c$ & Q & $1 / 3$ & $2 / 3$ & $1 / 4$ & $0.0106(3)$ & $0.84(2)$ \\
\hline
\end{tabular}

Table 4 Atomic coordinates and displacement parameters $\left(\AA^{2}\right)$ for the substructure $\mathrm{V}_{12} \mathrm{Sn}\left(h P 26, P 6_{3} / m m c\right.$, $a=4.9433(5), c=17.935(3) \AA)$.

\begin{tabular}{c|c|c|c|c|c|c}
\hline Site & $\begin{array}{c}\text { Wyckoff } \\
\text { position }\end{array}$ & $\begin{array}{c}\text { Polyhedron } \\
\text { type }\end{array}$ & $x$ & $y$ & $z$ & $U_{\text {eq }}$ \\
\hline $\mathrm{V} 1$ & $4 f$ & $\mathrm{X}$ & $1 / 3$ & $2 / 3$ & $0.52253(6)$ & $0.0119(3)$ \\
\hline $\mathrm{V} 2$ & $4 e$ & $\mathrm{R}$ & 0 & 0 & $0.1781(11)$ & $0.0122(3)$ \\
\hline $\mathrm{V} 3$ & $2 d$ & $\mathrm{Q}$ & $1 / 3$ & $2 / 3$ & $3 / 4$ & $0.0088(4)$ \\
\hline $\mathrm{V} 4$ & $12 k$ & $\mathrm{X}$ & $0.505(2)$ & $0.010(4)$ & $0.6157(6)$ & $0.0097(2)$ \\
\hline $\mathrm{V} 5$ & $2 a$ & 20 -vertex & 0 & 0 & 0 & $0.0181(10)$ \\
\hline Sn6 & $2 c$ & $\mathrm{Q}$ & $1 / 3$ & $2 / 3$ & $1 / 4$ & $0.0106(3)$ \\
\hline
\end{tabular}


Table 5 Selected interatomic distances for the two substructures in $\mathrm{Nb}_{4.4} \mathrm{~V}_{1.6} \mathrm{Ni}_{6} \mathrm{Sn}_{0.9}$.

\begin{tabular}{|c|c|c|c|c|c|}
\hline \multicolumn{3}{|c|}{$\mathrm{Nb}_{5} \mathrm{Ni}_{6.84} \mathrm{~V}_{0.16} \mathrm{Sn}_{0.84}$} & \multicolumn{3}{|c|}{$\mathrm{V}_{12} \mathrm{Sn}$} \\
\hline \multicolumn{2}{|c|}{ Atoms } & \multirow{2}{*}{$\begin{array}{c}\delta, \AA \\
2.8002(18)\end{array}$} & \multicolumn{2}{|c|}{ Atoms } & \multirow{2}{*}{$\frac{\delta, \AA}{2.227(13)}$} \\
\hline $\mathrm{Nb} 1$ & $-1 \mathrm{Nb} 2$ & & \multirow[t]{5}{*}{ V1 } & $-3 \mathrm{~V} 4$ & \\
\hline & $-3 \mathrm{Ni} 4$ & $2.8240(16)$ & & $-3 \mathrm{~V} 4$ & $2.837(12)$ \\
\hline & $-3 \mathrm{Ni} 5 / \mathrm{V} 5$ & $2.8825(3)$ & & $-3 \mathrm{~V} 5$ & $2.8825(3)$ \\
\hline & $-6 \mathrm{Ni} 4$ & $2.9620(9)$ & & $-3 \mathrm{~V} 1$ & $2.9662(7)$ \\
\hline & $-3 \mathrm{Nb} 1$ & $2.9662(7)$ & & & \\
\hline \multirow[t]{5}{*}{$\mathrm{Nb} 2$} & $-1 \mathrm{Nb} 2$ & $2.559(2)$ & \multirow[t]{5}{*}{$\mathrm{V} 2$} & $-1 \mathrm{~V} 2$ & $2.58(4)$ \\
\hline & $-6 \mathrm{Ni} 4$ & $2.7337(7)$ & & $-6 \mathrm{~V} 4$ & $2.714(8)$ \\
\hline & $-1 \mathrm{Nb} 1$ & $2.8002(18)$ & & $-3 V 3$ & $3.132(8)$ \\
\hline & $-3 \operatorname{Sn} 6$ & $3.1277(6)$ & & $-3 \operatorname{Sn} 6$ & $3.132(8)$ \\
\hline & $-3 \mathrm{Nb3}$ & $3.1277(6)$ & & $-1 \mathrm{~V} 5$ & $3.194(19)$ \\
\hline \multirow[t]{3}{*}{ Nb3 } & $-6 \mathrm{Ni} 4$ & $2.8359(13)$ & \multirow[t]{3}{*}{ V3 } & $-6 \mathrm{~V} 4$ & $2.824(12)$ \\
\hline & $-3 \operatorname{Sn} 6$ & $2.8540(3)$ & & $-3 \operatorname{Sn} 6$ & $2.8540(3)$ \\
\hline & $-6 \mathrm{Nb} 2$ & $3.1277(6)$ & & $-6 \mathrm{~V} 2$ & $3.132(8)$ \\
\hline \multirow[t]{8}{*}{$\mathrm{Ni4}$} & $-2 \mathrm{Ni} 4$ & $2.462(3)$ & \multirow[t]{8}{*}{ V4 } & $-2 \mathrm{~V} 1$ & $2.227(13)$ \\
\hline & $-2 \mathrm{Ni} 4$ & $2.481(3)$ & & $-2 \mathrm{~V} 4$ & $2.39(3)$ \\
\hline & $-1 \mathrm{Ni5/V5}$ & $2.4897(14)$ & & $-2 \mathrm{~V} 4$ & $2.55(3)$ \\
\hline & $-2 \mathrm{Nb} 2$ & $2.7337(7)$ & & $-1 \mathrm{~V} 2$ & $2.714(8)$ \\
\hline & $-1 \mathrm{Nb} 1$ & $2.8240(16)$ & & -1 Sn6 & $2.777(12)$ \\
\hline & $-1 \operatorname{Sn} 6$ & $2.8303(13)$ & & $-1 \mathrm{~V} 3$ & $2.824(12)$ \\
\hline & $-1 \mathrm{Nb3}$ & $2.8359(13)$ & & $-1 \mathrm{~V} 1$ & $2.837(12)$ \\
\hline & $-2 \mathrm{Nb} 1$ & $2.9620(9)$ & & $-2 \mathrm{~V} 5$ & $3.227(6)$ \\
\hline \multirow[t]{3}{*}{ Ni5/V5 } & $-6 \mathrm{Ni} 4$ & $2.4897(14)$ & \multirow[t]{3}{*}{ V5 } & $-6 \mathrm{V1}$ & $2.8825(3)$ \\
\hline & $-6 \mathrm{Nb} 1$ & $2.8825(3)$ & & $-2 \mathrm{~V} 2$ & $3.194(19)$ \\
\hline & & & & $-12 \mathrm{~V} 4$ & $3.227(6)$ \\
\hline \multirow[t]{3}{*}{ Sn6 } & $-6 \mathrm{Ni} 4$ & $2.8303(13)$ & \multirow[t]{3}{*}{ Sn6 } & $-6 \mathrm{~V} 4$ & $2.777(12)$ \\
\hline & $-3 \mathrm{Nb3}$ & $2.8540(3)$ & & $-3 \mathrm{~V} 3$ & $2.8540(3)$ \\
\hline & $-6 \mathrm{Nb} 2$ & $3.1277(6)$ & & $-6 \mathrm{~V} 2$ & $3.132(8)$ \\
\hline
\end{tabular}

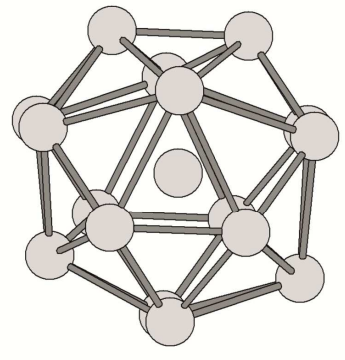

P

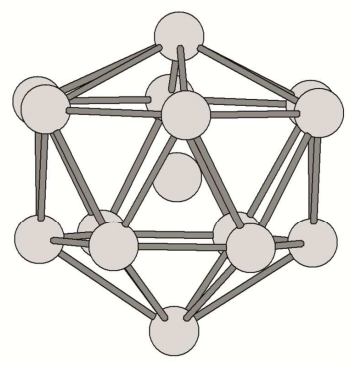

R

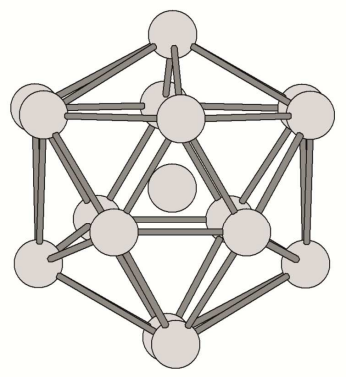

Q

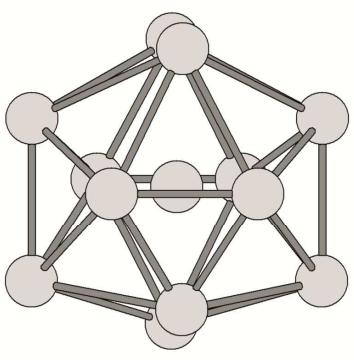

X

Fig. 1 The four Frank-Kasper polyhedra. 
Table 6 Structure types built from Frank-Kasper polyhedra: number of formula units (Z), number of $n$-vertex polyhedra per formula unit $\left(N_{n}\right)$, coefficients of the formula $\left(\mathrm{R}_{3} \mathrm{X}\right)_{i}\left(\mathrm{PX}_{2}\right)_{j}\left(\mathrm{Q}_{2} \mathrm{R}_{2} \mathrm{X}_{3}\right)_{k}$, average coordination number $(\langle C N\rangle)$.

\begin{tabular}{|c|c|c|c|c|c|c|c|c|c|c|c|c|}
\hline Structure type $^{\mathrm{a}}$ & $\begin{array}{l}\text { Pearson } \\
\text { symbol }\end{array}$ & $\begin{array}{l}\text { Space } \\
\text { group }\end{array}$ & $Z^{\mathrm{b}}$ & $N_{16}{ }^{\mathrm{c}}$ & $N_{15}{ }^{\mathrm{c}}$ & $N_{14}{ }^{\mathrm{c}}$ & $N_{12}{ }^{\mathrm{c}}$ & $i\left(\mathrm{R}_{3} \mathrm{X}\right)$ & $j\left(\mathrm{PX}_{2}\right)$ & $k\left(\mathrm{Q}_{2} \mathrm{R}_{2} \mathrm{X}_{3}\right)$ & $\begin{array}{c}\text { Basic } \\
\text { formula }^{\mathrm{d}}\end{array}$ & $\langle C N\rangle^{\mathrm{e}}$ \\
\hline $\mathrm{Cr} \mathbf{t f}, \mathrm{Cr}_{3} \mathrm{Si}$ & $c P 8$ & $P m-3 n$ & 2 & 0 & 0 & 3 & 1 & 1 & 0 & 0 & $\mathrm{~A}_{3} \mathrm{X}$ & 13.500 \\
\hline $\mathrm{W}(\mathrm{Al}, \mathrm{W})_{6} \mathrm{Ge}$ & $c P 8$ & $P m-3$ & 2 & 0 & 0 & 3 & 1 & 1 & 0 & 0 & $\mathrm{~A}_{3} \mathrm{X}$ & 13.500 \\
\hline$\beta$-Ta lt & $t P 30$ & $P-4$ & 2 & 0 & 2 & 8 & 5 & 2 & 0 & 1 & $\mathrm{~A}_{2} \mathrm{X}$ & 13.466 \\
\hline$\beta$-Ta rt & $t P 30$ & $P 4_{2} / m$ & 2 & 0 & 2 & 8 & 5 & 2 & 0 & 1 & $\mathrm{~A}_{2} \mathrm{X}$ & 13.466 \\
\hline $\mathbf{U}, \mathrm{Nb}_{2} \mathrm{Al}, \mathbf{C r}_{\mathbf{6}} \mathbf{F e}_{\mathbf{7}}$ & $t P 30$ & $P 4_{2} / m n m$ & 2 & 0 & 2 & 8 & 5 & 2 & 0 & 1 & $\mathrm{~A}_{2} \mathrm{X}$ & 13.466 \\
\hline$(\mathrm{Mo}, \mathrm{Cr})_{2}(\mathrm{Fe}, \mathrm{Cr}, \mathrm{Ni})_{13}$ & $m P 30$ & $P 2$ & 2 & 0 & 2 & 8 & 5 & 2 & 0 & 1 & $\mathrm{~A}_{2} \mathrm{X}$ & 13.466 \\
\hline$(\mathrm{Cr}, \mathrm{Fe}, \mathrm{Ni})$ & $o S 30$ & $\mathrm{Cmmm}$ & 2 & 0 & 2 & 8 & 5 & 2 & 0 & 1 & $\mathrm{~A}_{2} \mathrm{X}$ & 13.466 \\
\hline $\mathbf{K}_{\text {comp }}{ }^{\mathrm{f}}$ & $o P 82$ & Pmmm & 2 & 0 & 6 & 21 & 14 & 5 & 0 & 3 & $\mathrm{~A}_{14} \mathrm{X}_{27}$ & 13.463 \\
\hline$F_{\text {comp }}{ }^{f}$ & $h P 52$ & $P 6 / \mathrm{mmm}$ & 2 & 0 & 4 & 13 & 9 & 3 & 0 & 2 & $\mathrm{~A}_{9} \mathrm{X}_{17}$ & 13.462 \\
\hline $\mathbf{J}_{\text {comp }}{ }^{f}$ & $o P 22$ & Pmmm & 2 & 0 & 2 & 5 & 4 & 1 & 0 & 1 & $\mathrm{~A}_{4} \mathrm{X}_{7}$ & 13.455 \\
\hline $\mathrm{Mn}_{19}(\mathrm{Mn}, \mathrm{Si})_{10} \mathrm{Si}_{2}$ & $o I 186$ & Immm & 2 & 6 & 10 & 40 & 37 & 10 & 6 & 5 & $\mathrm{~A}_{56} \mathrm{X}_{37}$ & 13.441 \\
\hline $\mathrm{Zr}_{4} \mathrm{Al}_{3}$ & $h P 7$ & $P 6 / \mathrm{mmm}$ & 1 & 0 & 2 & 2 & 3 & 0 & 0 & 1 & $\mathrm{~A}_{4} \mathrm{X}_{3}$ & 13.428 \\
\hline $\mathrm{Mo}_{3}(\mathrm{Mo}, \mathrm{Cr})_{5}(\mathrm{Cr}, \mathrm{Ni})_{6}$ & $o P 56$ & Pnma & 4 & 1 & 2 & 5 & 6 & 1 & 1 & 1 & $\mathrm{~A}_{4} \mathrm{X}_{3}$ & 13.428 \\
\hline $\mathrm{Mo}_{3}(\mathrm{Mo}, \mathrm{Ni})_{5} \mathrm{Ni}_{6}$ & $o P 56$ & $P 2{ }_{1} 2_{1} 2_{1}$ & 4 & 1 & 2 & 5 & 6 & 1 & 1 & 1 & $\mathrm{~A}_{4} \mathrm{X}_{3}$ & 13.428 \\
\hline $\mathrm{Mn}_{5}(\mathrm{Mn}, \mathrm{Si}, \mathrm{Fe})_{6}$ & $m S 110$ & $C 2$ & 2 & 7 & 4 & 19 & 25 & 5 & 7 & 2 & $\mathrm{~A}_{6} \mathrm{X}_{5}$ & 13.418 \\
\hline $\mathrm{Mn}_{40}(\mathrm{Mn}, \mathrm{Si}, \mathrm{Fe})_{9} \mathrm{Si}_{6}$ & $m S 220$ & $C 2$ & 4 & 7 & 4 & 19 & 25 & 5 & 7 & 2 & $\mathrm{~A}_{6} \mathrm{X}_{5}$ & 13.418 \\
\hline $\mathrm{Mo}_{8}(\mathrm{Mo}, \mathrm{Cr}, \mathrm{Co})_{25}(\mathrm{Cr}, \mathrm{Co})_{20}$ & $h R 159$ & $R-3$ & 3 & 8 & 6 & 12 & 27 & 2 & 8 & 3 & $\mathrm{~A}_{26} \mathrm{X}_{27}$ & 13.396 \\
\hline $\begin{array}{c}\mathrm{Cs}_{6} \mathrm{~K}_{7}, \mathrm{Li}_{4} \mathrm{Cu}_{6}(\mathrm{Al}, \mathrm{Cu})_{3} \\
\mathrm{Nb}_{5} \mathrm{Ni}_{6.84} \mathrm{~V}_{0.16} \mathrm{Sn}_{0.84}{ }_{\mathrm{g}}\end{array}$ & $h P 26$ & $P 6_{3} / m m c$ & 2 & 2 & 2 & 2 & 7 & 0 & 2 & 1 & $\mathrm{~A}_{6} \mathrm{X}_{7}$ & 13.385 \\
\hline $\mathrm{Th}_{6} \mathrm{Cd}_{7}, \mathrm{~W}_{6}(\mathrm{Fe}, \mathrm{Si}, \mathrm{W})_{7}$ & $o P 26$ & Pbam & 2 & 2 & 2 & 2 & 7 & 0 & 2 & 1 & $\mathrm{~A}_{6} \mathrm{X}_{7}$ & 13.385 \\
\hline $\mathrm{W}_{6} \mathrm{Fe}_{7}$ & $h R 39$ & $R-3 m$ & 3 & 2 & 2 & 2 & 7 & 0 & 2 & 1 & $\mathrm{~A}_{6} \mathrm{X}_{7}$ & 13.385 \\
\hline $\mathrm{Nb}_{6}(\mathrm{Ni}, \mathrm{Al}, \mathrm{Nb})_{7}$ & $o P 52$ & Pnma & 4 & 2 & 2 & 2 & 7 & 0 & 2 & 1 & $\mathrm{~A}_{6} \mathrm{X}_{7}$ & 13.385 \\
\hline $\begin{array}{l}\mathbf{B e}_{15}(\mathrm{Be}, \mathbf{R h})_{2} \mathbf{R h}_{2} \\
\mathrm{Mg}_{4} \mathbf{Z n}_{10}(\mathrm{Zn}, \mathbf{A l})_{2}\end{array}$ & $h P 19$ & $P-6 m 2$ & 1 & 4 & 2 & 2 & 11 & 0 & 4 & 1 & $\mathrm{~A}_{8} \mathrm{X}_{11}$ & 13.369 \\
\hline $\mathrm{V}_{8}(\mathrm{Ni}, \mathrm{Si})_{11}$ & $m S 228$ & $C c$ & 12 & 4 & 2 & 2 & 11 & 0 & 4 & 1 & $\mathrm{~A}_{8} \mathrm{X}_{11}$ & 13.369 \\
\hline $\mathrm{Mog}_{9} \mathrm{Fe}_{13}$ & $m S 88$ & $C 2 / m$ & 4 & 5 & 2 & 2 & 13 & 0 & 5 & 1 & $\mathrm{~A}_{9} \mathrm{X}_{13}$ & 13.364 \\
\hline $\mathrm{V}_{2}(\mathrm{Co}, \mathrm{Si})_{3}$ & $m S 50$ & $C 2 / m$ & 2 & 6 & 2 & 2 & 15 & 0 & 6 & 1 & $\mathrm{~A}_{2} \mathrm{X}_{3}$ & 13.360 \\
\hline $\begin{array}{c}\mathrm{Mg}_{32}(\mathrm{Zn}, \mathrm{Al})_{49}, \\
\mathbf{N a}_{\mathbf{2 6}}(\mathbf{N a}, \mathbf{A u})_{\mathbf{6}} \mathbf{A} \mathbf{u}_{\mathbf{3 7}} \mathbf{S n}_{\mathbf{1 2}}\end{array}$ & $c I 162$ & Im-3 & 2 & 20 & 6 & 6 & 49 & 0 & 20 & 3 & $\mathrm{~A}_{32} \mathrm{X}_{49}$ & 13.358 \\
\hline $\mathrm{Mn}_{14}(\mathrm{Co}, \mathrm{Si}, \mathrm{Mn})_{23}$ & $o P 74$ & Pnnm & 2 & 10 & 2 & 2 & 23 & 0 & 10 & 1 & $\mathrm{~A}_{14} \mathrm{X}_{23}$ & 13.351 \\
\hline $\mathrm{Mg}_{4} \mathrm{Zn}_{7}$ & $m S 110$ & $C 2 / m$ & 2 & 16 & 2 & 2 & 35 & 0 & 16 & 1 & $\mathrm{~A}_{4} \mathrm{X}_{7}$ & 13.345 \\
\hline $\mathrm{MgZn}_{2}$ & $h P 12$ & $P 6_{3} / m m c$ & 4 & 1 & 0 & 0 & 2 & 0 & 1 & 0 & $\mathrm{AX}_{2}$ & 13.333 \\
\hline $\mathrm{MgCu}_{2}$ & $c F 24$ & $F d-3 m$ & 8 & 1 & 0 & 0 & 2 & 0 & 1 & 0 & $\mathrm{AX}_{2}$ & 13.333 \\
\hline
\end{tabular}

${ }^{\mathrm{a}}$ Structure types not mentioned in [4] are marked in bold. ${ }^{\mathrm{b}} \mathrm{Z}$ is the largest common divisor. ${ }^{\mathrm{c}} N_{16}, N_{15}, N_{14}$, and $N_{12}$ are the numbers of respectively 16-, 15-, 14-, and 12-vertex polyhedra in the unit cell normalized by $Z$. ${ }^{\mathrm{d}} \mathrm{A}=\mathrm{R}+\mathrm{Q}+\mathrm{P} .{ }^{\mathrm{e}}$ Weight-averaged coordination number. ${ }^{\mathrm{f}}$ The phases were found in a multicomponent Ni-based alloy containing $\mathrm{Cr}$, Mo, W, Fe, Si, Al, Ti, Mn, Zr, Ce, C, and B [15]; EDX analysis of the phase $\mathbf{F}_{\text {comp }}$ revealed the following composition (in at.\%): Mo 45.1, $\mathrm{Cr} 23.7, \mathrm{Ni} 19.9$, and $\mathrm{W} 11.3$. ${ }^{\mathrm{g}}$ Substructure of the compound $\mathrm{Nb}_{4.4} \mathrm{~V}_{1.6} \mathrm{Ni}_{6} \mathrm{Sn}_{0.9}$.

Eq. 8 can be rewritten in the following way:

$N_{12}=2 \cdot N_{16}+7 / 6 \cdot N_{15}+1 / 3 \cdot N_{14}$.

Yarmolyuk and Kripyakevich [4] established the following equations:

$\langle C N\rangle=13.6-0.4 \cdot C_{12}$

and

$C_{14}-C_{16}=1.4-2.6 \cdot C_{12}$, where $\langle C N\rangle$ is the average coordination number $\left.(<C N\rangle=16 \cdot C_{16}+15 \cdot C_{15}+14 \cdot C_{14}+12 \cdot C_{12}\right), C_{16}, C_{15}$, $C_{14}$, and $C_{12}$ are the fractions of the corresponding polyhedra in the structure $\left(C_{16}+C_{15}+C_{14}+C_{12}=1\right.$ and e.g. $C_{12}=\frac{N_{12}}{N_{16}+N_{15}+N_{14}+N_{12}}$ ). 


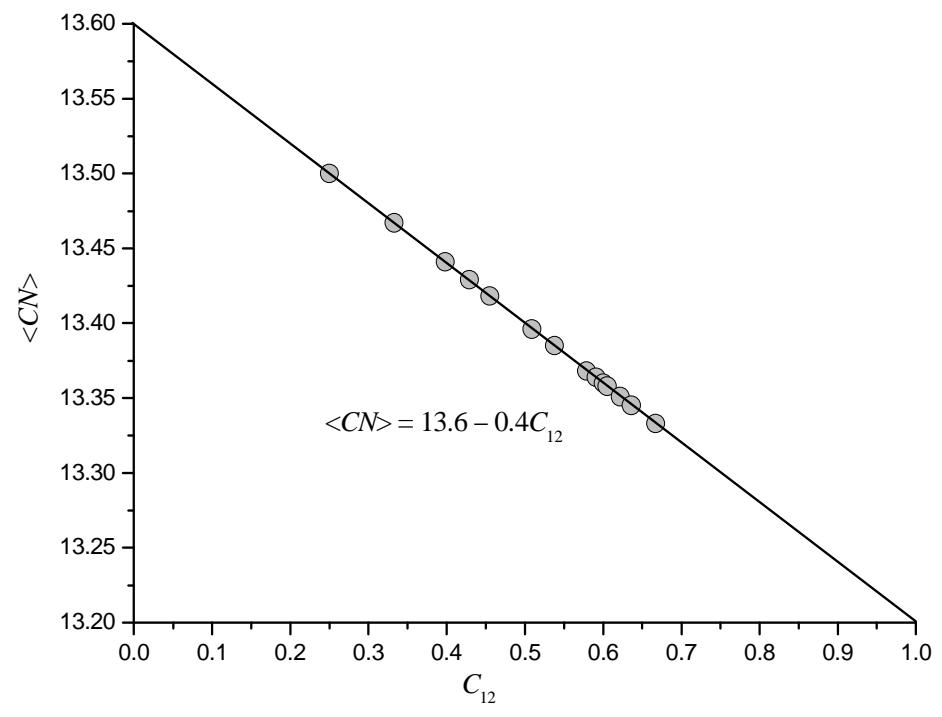

Fig. 2 Average coordination number versus icosahedron content for t.c.p. structures.

Fig. 2 shows a plot of Eq. 11 including all t.c.p. structures known so far. It is possible to transform Eq. 10 into

$$
\begin{aligned}
& \frac{N_{12}}{N_{16}+N_{15}+N_{14}+N_{12}}=\frac{2 \cdot N_{16}}{N_{16}+N_{15}+N_{14}+N_{12}}+ \\
& +\frac{7 / 6 \cdot N_{15}}{N_{16}+N_{15}+N_{14}+N_{12}}+\frac{1 / 3 \cdot N_{14}}{N_{16}+N_{15}+N_{14}+N_{12}}
\end{aligned}
$$
or

$C_{12}=2 \cdot C_{16}+7 / 6 \cdot C_{15}+1 / 3 \cdot C_{14}$

When $C_{15}$ is replaced by $C_{15}=1-C_{16}-C_{14}-C_{12}$ then $C_{12}=2 \cdot C_{16}+7 / 6 \cdot\left(1-C_{16}-C_{14}-C_{12}\right)+1 / 3 \cdot C_{14}$ and $C_{14}-C_{16}=1.4-2.6 \cdot C_{12}$ (i.e. Eq. 12). Subtracting the latter equation from the equation $C_{16}+C_{15}+C_{14}=$ $1-C_{12}$ multiplied by $15 \cdot$ one obtains: $15 \cdot C_{16}+15 \cdot C_{15}$ $+15 \cdot C_{14}-C_{14}+C_{16}=15-15 \cdot C_{12}-1.4+2.6 C_{12}$ and $16 \cdot C_{16}+15 \cdot C_{15}+14 \cdot C_{14}+12 \cdot C_{12}=13.6-0.4 \cdot C_{12}$ and $\langle C N\rangle=13.6-0.4 \cdot C_{12}$ (i.e. Eq. 11$)$.

Recently Sikirić et al. [16], using special software, derived hypothetical structures with Frank-Kasper polyhedra. They confirmed 22 known structures and obtained 71 new structures with $N_{12} \leq 11$. Among them are three combinations of polyhedra, $\mathrm{P}_{2} \mathrm{Q}_{2} \mathrm{R}_{4} \mathrm{X}_{7}$, $\mathrm{P}_{0} \mathrm{Q}_{2} \mathrm{R}_{3} \mathrm{X}_{3}$, and $\mathrm{P}_{0} \mathrm{Q}_{2} \mathrm{R}_{4} \mathrm{X}_{3}$, which are not allowed according to the Yarmolyuk-Kripyakevich criterion, and two combinations, $\mathrm{P}_{1} \mathrm{Q}_{0} \mathrm{R}_{3} \mathrm{X}_{3}$ and $\mathrm{P}_{2} \mathrm{Q}_{0} \mathrm{R}_{3} \mathrm{X}_{5}$, which do not follow the condition (if both $i \geq 1$ and $j \geq 1$, then also $k \geq 1$ ) formulated by Hellner and Pearson [17].

Let us look at the "Yarmolyuk-Kripyakevich criterion" in a slightly different way. As can be seen from Table 6, 18 different combinations of $\mathrm{R}_{3} \mathrm{X}, \mathrm{PX}_{2}$, and $\mathrm{Q}_{2} \mathrm{R}_{2} \mathrm{X}_{3}$ prototypes are known as of today and some of them are represented by several structure types. The combination $\mathrm{P}_{0} \mathrm{Q}_{0} \mathrm{R}_{3} \mathrm{X}_{1}\left(N_{12}=1\right)$ appears twice: with the space groups $P m-3 n$ and $P m-3$ (ordering variant with lower symmetry), $\mathrm{P}_{1} \mathrm{Q}_{0} \mathrm{R}_{0} \mathrm{X}_{2}$
$\left(N_{12}=2\right)$ appears twice: $P 6_{3} / m m c$ and $F d-3 m$ (stacking variants), $\mathrm{P}_{0} \mathrm{Q}_{2} \mathrm{R}_{8} \mathrm{X}_{5}\left(N_{12}=5\right)$ appears five times: $P-4$, $\mathrm{P}_{2} / \mathrm{m}, \mathrm{P}_{2} / \mathrm{mnm}, \mathrm{P} 2$, and Cmmm, $\mathrm{P}_{1} \mathrm{Q}_{2} \mathrm{R}_{5} \mathrm{X}_{6}\left(N_{12}=6\right)$ appears twice: Pnma and $P 2_{1} 2_{1} 2_{1}$, and $\mathrm{P}_{2} \mathrm{Q}_{2} \mathrm{R}_{2} \mathrm{X}_{7}$ $\left(N_{12}=7\right)$ four times: $P 6_{3} / m m c$, Pbam, $R-3 m$, and Pnma, $\mathrm{P}_{4} \mathrm{Q}_{2} \mathrm{R}_{2} \mathrm{X}_{11}\left(N_{12}=11\right)$ appears twice: $P-6 m 2$ and $C c$, and $\mathrm{P}_{7} \mathrm{Q}_{4} \mathrm{R}_{19} \mathrm{X}_{25}\left(N_{12}=25\right)$ twice: both times with space group $C 2$. The other combinations have only one representative. As can be seen from Table 7, for each value of $N_{12}$ (except for $N_{12}=1,2$, and 4), theoretically there exist several possible combinations of $\mathrm{R}_{3} \mathrm{X}, \mathrm{PX}_{2}$, and $\mathrm{Q}_{2} \mathrm{R}_{2} \mathrm{X}_{3}$, however, only one combination is observed. Thus, a question arises: why does a particular combination form among a number of different possible variants. Let us return to Eq. 10: $N_{12}=2 \cdot N_{16}+7 / 6 \cdot N_{15}+1 / 3 \cdot N_{14}$; the number of solutions increases with increasing $N_{12}$. Considering that $N_{16}, N_{15}, N_{14}, N_{12}$ are integers, at least one of them is odd, and $N_{14} \geq N_{15}$, for the limiting values $N_{16}, N_{15}$, $N_{14}, N_{12}=50$ we found 3475 solutions, part of which are presented in Table 7 . As can be seen, for $N_{12}=1$ and 2 one single combination is found: $\mathrm{P}_{0} \mathrm{Q}_{0} \mathrm{R}_{3} \mathrm{X}_{1}$ $\left(\mathrm{Cr}_{3} \mathrm{Si}\right)$ and $\mathrm{P}_{1} \mathrm{Q}_{0} \mathrm{R}_{0} \mathrm{X}_{2}\left(\mathrm{MgZn}_{2}\right)$, but for $N_{12}=3$ two variants are possible: $\mathrm{P}_{0} \mathrm{Q}_{2} \mathrm{R}_{2} \mathrm{X}_{3}=\mathrm{Q}_{2} \mathrm{R}_{2} \mathrm{X}_{3}$ and $\mathrm{P}_{1} \mathrm{Q}_{0} \mathrm{R}_{3} \mathrm{X}_{3}=\mathrm{PX}_{2}+\mathrm{R}_{3} \mathrm{X}$, combining different parent structures, but having the same average coordination number $\langle\mathrm{CN}\rangle=13.429$. The prototype $\mathrm{Zr}_{4} \mathrm{Al}_{3}$ belongs to the first variant, $\mathrm{Q}_{2} \mathrm{R}_{2} \mathrm{X}_{3}$, i.e. represents the combination that contains Q-polyhedra. The next known structures in Table 7 (shaded rows) are also combinations of $\left(\mathrm{R}_{3} \mathrm{X}\right)_{i}\left(\mathrm{PX}_{2}\right)_{j}\left(\mathrm{Q}_{2} \mathrm{R}_{2} \mathrm{X}_{3}\right)_{k}$ including $\mathrm{Q}_{2} \mathrm{R}_{2} \mathrm{X}_{3}$ fragments. This fact was observed by Hellner and Pearson [17] (if both $i \geq 1$ and $j \geq 1$, then $k \geq 1$ ). When Hellner and Pearson considered different possible cases of generating hypothetical structures containing P-, R- and X-polyhedra without Q-polyhedra, they found that the resulting structures contained not only Frank-Kasper polyhedra, 
Table 7 Solutions to Eq. 10, ordered according to increasing number of icosahedral coordination per formula unit $\left(N_{12}\right)$. Polyhedron distributions observed in known structures are shadowed (see Table 6 for explanations of codes).

\begin{tabular}{|c|c|c|c|c|c|c|c|c|c|c|c|c|c|c|c|}
\hline$N_{12}$ & $N_{14}$ & $N_{15}$ & $N_{16}$ & $i\left(\mathrm{R}_{3} \mathrm{X}\right)$ & $j\left(\mathrm{PX}_{2}\right)$ & $k\left(\mathrm{Q}_{2} \mathrm{R}_{2} \mathrm{X}_{3}\right)$ & $\langle C N\rangle$ & $N_{12}$ & $N_{14}$ & $N_{15}$ & $N_{16}$ & $i\left(\mathrm{R}_{3} \mathrm{X}\right)$ & $j\left(\mathrm{PX}_{2}\right)$ & $k\left(\mathrm{Q}_{2} \mathrm{R}_{2} \mathrm{X}_{3}\right)$ & $\langle C N\rangle$ \\
\hline 1 & 3 & 0 & 0 & 1 & 0 & 0 & 13.500 & 13 & 18 & 6 & 0 & 4 & 0 & 3 & 13.459 \\
\hline 2 & 0 & 0 & 1 & 0 & 1 & 0 & 13.333 & 13 & 19 & 4 & 1 & 5 & 1 & 2 & 13.459 \\
\hline 3 & 2 & 2 & 0 & 0 & 0 & 1 & 13.429 & 13 & 20 & 2 & 2 & 6 & 2 & 1 & 13.459 \\
\hline 3 & 3 & 0 & 1 & 1 & 1 & 0 & 13.429 & 13 & 25 & 4 & 0 & 7 & 0 & 2 & 13.476 \\
\hline 4 & 5 & 2 & 0 & 1 & 0 & 1 & 13.455 & 13 & 26 & 2 & 1 & 8 & 1 & 1 & 13.476 \\
\hline 5 & 2 & 2 & 1 & 0 & 1 & 1 & 13.400 & 13 & 32 & 2 & 0 & 10 & 0 & 1 & 13.489 \\
\hline 5 & 8 & 2 & 0 & 2 & 0 & 1 & 13.467 & 14 & 5 & 2 & 5 & 1 & 5 & 1 & 13.385 \\
\hline 6 & 5 & 2 & 1 & 1 & 1 & 1 & 13.429 & 14 & 8 & 8 & 1 & 0 & 1 & 4 & 13.419 \\
\hline 6 & 11 & 2 & 0 & 3 & 0 & 1 & 13.474 & 14 & 9 & 6 & 2 & 1 & 2 & 3 & 13.419 \\
\hline 7 & 2 & 2 & 2 & 0 & 2 & 1 & 13.385 & 14 & 10 & 4 & 3 & 2 & 3 & 2 & 13.419 \\
\hline 7 & 7 & 4 & 0 & 1 & 0 & 2 & 13.444 & 14 & 11 & 2 & 4 & 3 & 4 & 1 & 13.419 \\
\hline 7 & 8 & 2 & 1 & 2 & 1 & 1 & 13.444 & 14 & 15 & 6 & 1 & 3 & 1 & 3 & 13.444 \\
\hline 7 & 14 & 2 & 0 & 4 & 0 & 1 & 13.478 & 14 & 17 & 2 & 3 & 5 & 3 & 1 & 13.444 \\
\hline 8 & 4 & 4 & 1 & 0 & 1 & 2 & 13.412 & 14 & 21 & 6 & 0 & 5 & 0 & 3 & 13.463 \\
\hline 8 & 5 & 2 & 2 & 1 & 2 & 1 & 13.412 & 14 & 22 & 4 & 1 & 6 & 1 & 2 & 13.463 \\
\hline 8 & 11 & 2 & 1 & 3 & 1 & 1 & 13.455 & 14 & 23 & 2 & 2 & 7 & 2 & 1 & 13.463 \\
\hline 8 & 17 & 2 & 0 & 5 & 0 & 1 & 13.481 & 14 & 29 & 2 & 1 & 9 & 1 & 1 & 13.478 \\
\hline 9 & 2 & 2 & 3 & 0 & 3 & 1 & 13.375 & 14 & 35 & 2 & 0 & 11 & 0 & 1 & 13.490 \\
\hline 9 & 7 & 4 & 1 & 1 & 1 & 2 & 13.429 & 15 & 2 & 2 & 6 & 0 & 6 & 1 & 13.360 \\
\hline 9 & 8 & 2 & 2 & 2 & 2 & 1 & 13.429 & $\ldots$ & $\ldots$ & $\ldots$ & $\ldots$ & $\ldots$ & $\ldots$ & $\ldots$ & $\ldots$ \\
\hline 9 & 13 & 4 & 0 & 3 & 0 & 2 & 13.462 & 15 & 38 & 2 & 0 & 12 & 0 & 1 & 13.491 \\
\hline 9 & 14 & 2 & 1 & 4 & 1 & 1 & 13.462 & $\ldots$ & $\ldots$ & $\ldots$ & $\ldots$ & $\ldots$ & $\ldots$ & $\ldots$ & $\ldots$ \\
\hline 9 & 20 & 2 & 0 & 6 & 0 & 1 & 13.484 & 23 & 2 & 2 & 10 & 0 & 10 & 1 & 13.351 \\
\hline 10 & 5 & 2 & 3 & 1 & 3 & 1 & 13.400 & $\ldots$ & $\ldots$ & $\ldots$ & $\ldots$ & $\ldots$ & $\ldots$ & $\ldots$ & \\
\hline 10 & 9 & 6 & 0 & 1 & 0 & 3 & 13.440 & 23 & 50 & 2 & 2 & 16 & 2 & 1 & 13.481 \\
\hline 10 & 10 & 4 & 1 & 2 & 1 & 2 & 13.440 & $\ldots$ & $\ldots$ & $\ldots$ & $\ldots$ & $\ldots$ & $\ldots$ & $\ldots$ & $\ldots$ \\
\hline 10 & 11 & 2 & 2 & 3 & 2 & 1 & 13.440 & 25 & 2 & 2 & 11 & 0 & 11 & 1 & 13.350 \\
\hline 10 & 17 & 2 & 1 & 5 & 1 & 1 & 13.467 & $\ldots$ & $\ldots$ & $\ldots$ & $\ldots$ & $\ldots$ & $\ldots$ & $\ldots$ & $\ldots$ \\
\hline 10 & 23 & 2 & 0 & 7 & 0 & 1 & 13.486 & 25 & 19 & 4 & 7 & 5 & 7 & 2 & 13.418 \\
\hline 11 & 2 & 2 & 4 & 0 & 4 & 1 & 13.368 & $\ldots$ & $\ldots$ & $\ldots$ & $\ldots$ & $\ldots$ & $\ldots$ & $\ldots$ & $\ldots$ \\
\hline 11 & 6 & 6 & 1 & 0 & 1 & 3 & 13.417 & 25 & 50 & 2 & 3 & 16 & 3 & 1 & 13.475 \\
\hline 11 & 7 & 4 & 2 & 1 & 2 & 2 & 13.417 & $\ldots$ & $\ldots$ & $\ldots$ & $\ldots$ & $\ldots$ & $\ldots$ & $\ldots$ & $\ldots$ \\
\hline 11 & 8 & 2 & 3 & 2 & 3 & 1 & 13.417 & 27 & 2 & 2 & 12 & 0 & 12 & 1 & 13.349 \\
\hline 11 & 12 & 6 & 0 & 2 & 0 & 3 & 13.448 & $\ldots$ & $\ldots$ & $\ldots$ & $\ldots$ & $\ldots$ & $\ldots$ & $\ldots$ & $\ldots$ \\
\hline 11 & 13 & 4 & 1 & 3 & 1 & 2 & 13.448 & 27 & 12 & 6 & 8 & 2 & 8 & 3 & 13.396 \\
\hline 11 & 14 & 2 & 2 & 4 & 2 & 1 & 13.448 & $\ldots$ & $\ldots$ & $\ldots$ & $\ldots$ & $\ldots$ & $\ldots$ & $\ldots$ & $\ldots$ \\
\hline 11 & 19 & 4 & 0 & 5 & 0 & 2 & 13.471 & 27 & 50 & 2 & 4 & 16 & 4 & 1 & 13.470 \\
\hline 11 & 20 & 2 & 1 & 6 & 1 & 1 & 13.471 & $\ldots$ & $\ldots$ & $\ldots$ & $\ldots$ & $\ldots$ & $\ldots$ & $\ldots$ & $\ldots$ \\
\hline 11 & 26 & 2 & 0 & 8 & 0 & 1 & 13.487 & 35 & 2 & 2 & 16 & 0 & 16 & 1 & 13.345 \\
\hline 12 & 4 & 4 & 3 & 0 & 3 & 2 & 13.391 & $\ldots$ & $\ldots$ & $\ldots$ & $\ldots$ & $\ldots$ & $\ldots$ & $\ldots$ & $\ldots$ \\
\hline 12 & 5 & 2 & 4 & 1 & 4 & 1 & 13.391 & 35 & 50 & 14 & 1 & 12 & 1 & 7 & 13.460 \\
\hline 12 & 9 & 6 & 1 & 1 & 1 & 3 & 13.429 & $\ldots$ & $\ldots$ & $\ldots$ & $\ldots$ & $\ldots$ & $\ldots$ & $\ldots$ & $\ldots$ \\
\hline 12 & 11 & 2 & 3 & 3 & 3 & 1 & 13.429 & 37 & 2 & 2 & 17 & 0 & 17 & 1 & 13.345 \\
\hline 12 & 16 & 4 & 1 & 4 & 1 & 2 & 13.455 & $\ldots$ & $\ldots$ & $\ldots$ & $\ldots$ & $\ldots$ & $\ldots$ & $\ldots$ & $\ldots$ \\
\hline 12 & 17 & 2 & 2 & 5 & 2 & 1 & 13.455 & 37 & 40 & 10 & 6 & 10 & 6 & 5 & 13.441 \\
\hline 12 & 23 & 2 & 1 & 7 & 1 & 1 & 13.474 & $\ldots$ & $\ldots$ & $\ldots$ & $\ldots$ & $\ldots$ & $\ldots$ & $\ldots$ & $\ldots$ \\
\hline 12 & 29 & 2 & 0 & 9 & 0 & 1 & 13.488 & 37 & 50 & 2 & 9 & 16 & 9 & 1 & 13.449 \\
\hline 13 & 2 & 2 & 5 & 0 & 5 & 1 & 13.364 & $\ldots$ & $\ldots$ & $\ldots$ & $\ldots$ & $\ldots$ & $\ldots$ & $\ldots$ & $\ldots$ \\
\hline 13 & 6 & 6 & 2 & 0 & 2 & 3 & 13.407 & 49 & 2 & 2 & 23 & 0 & 23 & 1 & 13.342 \\
\hline 13 & 7 & 4 & 3 & 1 & 3 & 2 & 13.407 & 49 & 6 & 6 & 20 & 0 & 20 & 3 & 13.358 \\
\hline 13 & 8 & 2 & 4 & 2 & 4 & 1 & 13.407 & $\ldots$ & $\ldots$ & $\ldots$ & $\ldots$ & $\ldots$ & $\ldots$ & $\ldots$ & $\ldots$ \\
\hline 13 & 11 & 8 & 0 & 1 & 0 & 4 & 13.438 & 49 & 50 & 26 & 1 & 8 & 1 & 13 & 13.444 \\
\hline 13 & 12 & 6 & 1 & 2 & 1 & 3 & 13.438 & 50 & 5 & 2 & 23 & 1 & 23 & 1 & 13.350 \\
\hline 13 & 13 & 4 & 2 & 3 & 2 & 2 & 13.438 & $\ldots$ & $\ldots$ & $\ldots$ & $\ldots$ & $\ldots$ & $\ldots$ & $\ldots$ & $\ldots$ \\
\hline 13 & 14 & 2 & 3 & 4 & 3 & 1 & 13.438 & 50 & 49 & 22 & 4 & 9 & 4 & 11 & 13.440 \\
\hline
\end{tabular}


but also other types of polyhedron occurred, or the ratios of the longest and shortest edges exceeded 4/3, etc., i.e. not all the requirements for a t.c.p. structure were obeyed. In other words, hypothetical structures built by combination of $\mathrm{P}_{0} \mathrm{Q}_{0} \mathrm{R}_{3} \mathrm{X}_{1} \quad\left(\mathrm{Cr}_{3} \mathrm{Si}\right)$ and $\mathrm{P}_{1} \mathrm{Q}_{0} \mathrm{R}_{0} \mathrm{X}_{2}\left(\mathrm{MgZn}_{2}\right)$ fragments alone are not realistic and $\mathrm{Q}_{2} \mathrm{R}_{2} \mathrm{X}_{3} \quad\left(\mathrm{Zr}_{4} \mathrm{Al}_{3}\right)$ fragments are needed to "stabilize" the structures. Taking into consideration the condition that $\mathrm{Q}_{2} \mathrm{R}_{2} \mathrm{X}_{3}$ fragments must always be present, the set of solutions is reduced to 3162 (parent structures not included).

Counterexamples to the Yarmolyuk-Kripyakevich $\left(\mathrm{P}_{2} \mathrm{Q}_{2} \mathrm{R}_{4} \mathrm{X}_{7}, \quad \mathrm{P}_{0} \mathrm{Q}_{2} \mathrm{R}_{3} \mathrm{X}_{3}, \quad \mathrm{P}_{0} \mathrm{Q}_{2} \mathrm{R}_{4} \mathrm{X}_{3}\right)$ and HellnerPearson $\left(P_{1} Q_{0} R_{3} X_{3}\right.$ and $\left.P_{2} Q_{0} R_{3} X_{5}\right)$ rules were found in [16], which indicates that these rules express not all geometrical principles allowing the distribution of 16-, 15-, 14-, and 12-vertex polyhedra in space, but only part of them.

As can be seen from Table 7, the simplest combinations deduced from the YarmolyukKripyakevich criterion, having the lowest average coordination number and number of fragments $(i+j+k)$, are in most cases observed.

The simplest solutions of Eq. 10 considering the above-mentioned conditions are compiled in Table 8. Some likely "genealogical code" (repetition) is present after $N_{12}>4:(2,5,2,4)$ for $N_{14},(2,2,2,4)$ for $N_{15}$, and $(1+2 n, \underline{1+2 n}, \underline{1+2 n}+1, \underline{1+2 n})$ where $n=0,1,2, \ldots$ for $N_{16}$. The formulas to find the $\mathrm{P}_{j} \mathrm{Q}_{2 k} \mathrm{R}_{3 i+2 k} \mathrm{X}_{i+2 j+3 k}$ combinations can be expressed as: $\mathrm{P}_{1+2 n} \mathrm{Q}_{2} \mathrm{R}_{2} \mathrm{X}_{5+4 n}$, $\mathrm{P}_{1+2 n} \mathrm{Q}_{2} \mathrm{R}_{5} \mathrm{X}_{6+4 n}, \mathrm{P}_{1+2 n+1} \mathrm{Q}_{2} \mathrm{R}_{2} \mathrm{X}_{7+4 n}, \mathrm{P}_{1+2 n} \mathrm{Q}_{4} \mathrm{R}_{4} \mathrm{X}_{8+4 n}$.

It may be noted that no structure with $N_{12}$ has so far been observed, even if this icosahedron content corresponds to various alternative variant(s), some of them with the same $\langle C N\rangle$. This occurs for $\mathrm{P}_{1+2 n} \mathrm{Q}_{4} \mathrm{R}_{4} \mathrm{X}_{8+4 n}$, so this formula may not have a sense. Moreover, in the majority of the cases the simplest combinations without $\mathrm{R}_{3} \mathrm{X}$ fragments are observed for odd $N_{12}\left(\mathrm{P}_{1} \mathrm{Q}_{2} \mathrm{R}_{5} \mathrm{X}_{6}\right.$ constitutes an exception). They always contain equal numbers of Q- and R-polyhedra, and can be found from the formulas $\mathrm{P}_{1+2 n} \mathrm{Q}_{2} \mathrm{R}_{2} \mathrm{X}_{5+4 n}$ and $\mathrm{P}_{1+2 n+1} \mathrm{Q}_{2} \mathrm{R}_{2} \mathrm{X}_{7+4 n}$.

In the cases where $N_{12}=5$ and 25 the simplest combinations $\mathrm{P}_{1} \mathrm{Q}_{2} \mathrm{R}_{2} \mathrm{X}_{5}(\mathrm{AX})$ and $\mathrm{P}_{11} \mathrm{Q}_{2} \mathrm{R}_{2} \mathrm{X}_{25}\left(\mathrm{~A}_{3} \mathrm{X}_{5}\right)$ have not been observed, but instead the combinations $\mathrm{P}_{0} \mathrm{Q}_{2} \mathrm{R}_{8} \mathrm{X}_{5}\left(\mathrm{~A}_{2} \mathrm{X}\right)$ and $\mathrm{P}_{7} \mathrm{Q}_{4} \mathrm{R}_{19} \mathrm{X}_{25}\left(\mathrm{~A}_{6} \mathrm{X}_{5}\right)$ have been found. For $N_{12}=49$ the second simplest combination has been observed, whereas for $N_{12}=14,27,37$ the hitherto observed combinations are located in the middle of the list. It hence appears that additional factors act, together with the YarmolyukKripyakevich criterion, to determine the preferred combinations.

All experimentally observed combinations of $\left(\mathrm{R}_{3} \mathrm{X}\right)_{i}\left(\mathrm{PX}_{2}\right)_{j}\left(\mathrm{Q}_{2} \mathrm{R}_{2} \mathrm{X}_{3}\right)_{k}$ reported so far are compiled in Table 9. They have been subdivided into four groups, according to the fragments they contain. In group I are located combinations without $\mathrm{PX}_{2}$ fragments (only 12-, 14-, 15-vertex polyhedra are present) and the $\mathrm{R}_{3} \mathrm{X}$ $\left(\mathrm{Cr}_{3} \mathrm{Si}\right.$ ) parent structure. Groups II and III contain combinations without $\mathrm{R}_{3} \mathrm{X}$ fragment (equal numbers of Q- and R-polyhedra) and the $\mathrm{Q}_{2} \mathrm{R}_{2} \mathrm{X}_{3}\left(\mathrm{Zr}_{4} \mathrm{Al}_{3}\right)$ parent structure. Combinations of all three kinds of fragment are listed in group IV, together with the $\mathrm{PX}_{2}$ $\left(\mathrm{MgZn}_{2}\right)$ parent structure. Combinations that do not correspond to the simplest solutions of Eq. 10 for a particular value of $N_{12}$, i.e. which have larger average coordination number and/or not the minimal number of fragments $(i+j+k)$, are marked in italic. Such situations occur for groups I, III, and IV, while in group II all the observed structure types correspond to the simplest combinations deduced from the formula $\mathrm{P}_{1+2 n+1} \mathrm{Q}_{2} \mathrm{R}_{2} \mathrm{X}_{7+4 n}$ (see Table 8). It may be noted that in each group only certain values of $i, j$, and $k$ are allowed. These numbers form a series where every next number is the sum of the two preceding ones: $i, j$, $k \equiv 0,1,1,2,3,5,8, \ldots$, known as the Fibonacci sequence.

Table 8 The simplest solutions to Eq. 10. Distributions of polyhedra observed in known structures are shadowed.

\begin{tabular}{c|c|c|c|c|c|c|c|c|c|c|c}
\hline$N_{12}$ & $N_{14}$ & $N_{15}$ & $N_{16}$ & $N_{12}$ & $N_{14}$ & $N_{15}$ & $N_{16}$ & $N_{12}$ & $N_{14}$ & $N_{15}$ & $N_{16}$ \\
\hline 1 & 3 & 0 & 0 & 17 & 2 & 2 & 7 & 33 & 2 & 2 & 15 \\
2 & 0 & 0 & 1 & 18 & 5 & 2 & 7 & 34 & 5 & 2 & 15 \\
3 & 2 & 2 & 0 & 19 & 2 & 2 & 8 & 35 & 2 & 2 & 16 \\
4 & 5 & 2 & 0 & 20 & 4 & 4 & 7 & 36 & 4 & 4 & 15 \\
\hline 5 & 2 & 2 & 1 & 21 & 2 & 2 & 9 & 37 & 2 & 2 & 17 \\
6 & 5 & 2 & 1 & 22 & 5 & 2 & 9 & 38 & 5 & 2 & 17 \\
7 & 2 & 2 & 2 & 23 & 2 & 2 & 10 & 39 & 2 & 2 & 18 \\
8 & 4 & 4 & 1 & 24 & 4 & 4 & 9 & 40 & 4 & 4 & 17 \\
\hline 9 & 2 & 2 & 3 & 25 & 2 & 2 & 11 & 41 & 2 & 2 & 19 \\
10 & 5 & 2 & 3 & 26 & 5 & 2 & 11 & 42 & 5 & 2 & 19 \\
11 & 2 & 2 & 4 & 27 & 2 & 2 & 12 & 43 & 2 & 2 & 20 \\
12 & 4 & 4 & 3 & 28 & 4 & 4 & 11 & 44 & 4 & 4 & 19 \\
\hline 13 & 2 & 2 & 5 & 29 & 2 & 2 & 13 & 45 & 2 & 2 & 21 \\
14 & 5 & 2 & 5 & 30 & 5 & 2 & 13 & 46 & 5 & 2 & 21 \\
15 & 2 & 2 & 6 & 31 & 2 & 2 & 14 & 47 & 2 & 2 & 22 \\
16 & 4 & 4 & 5 & 32 & 4 & 4 & 13 & 48 & 4 & 4 & 21 \\
\hline
\end{tabular}


Table 9 Experimentally observed combinations $\left(\mathrm{R}_{3} \mathrm{X}\right)_{i}\left(\mathrm{PX}_{2}\right)_{j}\left(\mathrm{Q}_{2} \mathrm{R}_{2} \mathrm{X}_{3}\right)_{k}$, sorted according to the number of different fragments. Combinations that do not correspond to the simplest possible solutions are indicated in italics.

\begin{tabular}{|c|c|c|c|c|c|c|c|c|c|c|c|c|c|}
\hline \multicolumn{7}{|c|}{ Group I $(j=0):\left(\mathrm{R}_{3} \mathrm{X}\right)_{i}\left(\mathrm{Q}_{2} \mathrm{R}_{2} \mathrm{X}_{3}\right)_{k}$} & \multicolumn{7}{|c|}{ Group III $(i=0):\left(\mathrm{PX}_{2}\right)_{j}\left(\mathrm{Q}_{2} \mathrm{R}_{2} \mathrm{X}_{3}\right)_{k}$} \\
\hline$N_{12}$ & $N_{14}$ & $N_{15}$ & $N_{16}$ & $i\left(\mathrm{R}_{3} \mathrm{X}\right)$ & $j\left(\mathrm{PX}_{2}\right)$ & $k\left(\mathrm{Q}_{2} \mathrm{R}_{2} \mathrm{X}_{3}\right)$ & $N_{12}$ & $N_{14}$ & $N_{15}$ & $N_{16}$ & $i\left(\mathrm{R}_{3} \mathrm{X}\right)$ & $j\left(\mathrm{PX}_{2}\right)$ & $k\left(\mathrm{Q}_{2} \mathrm{R}_{2} \mathrm{X}_{3}\right)$ \\
\hline 1 & 3 & 0 & 0 & 1 & 0 & $\mathbf{0}$ & 3 & 2 & 2 & 0 & 0 & $20 \times 0$ & 2 \\
\hline 4 & 5 & 2 & 0 & 1 & 0 & 1 & 49 & 6 & 6 & 20 & 0 & $20 \times 1$ & 3 \\
\hline 5 & 8 & 2 & 0 & 2 & 0 & 1 & 11 & 2 & 2 & 4 & 0 & $20 \times 1$ & 5 \\
\hline 9 & 13 & 4 & 0 & 3 & 0 & 2 & 13 & 2 & 2 & 5 & 0 & $20 \times 2$ & 8 \\
\hline 14 & 21 & 6 & 0 & 5 & 0 & 3 & & & & & & & \\
\hline \multicolumn{7}{|c|}{ Group II $(i=0):\left(\mathrm{PX}_{2}\right)_{j}\left(\mathrm{Q}_{2} \mathrm{R}_{2} \mathrm{X}_{3}\right)_{k}$} & \multicolumn{7}{|c|}{ Group IV: $\left(\mathrm{R}_{3} \mathrm{X}\right)_{i}\left(\mathrm{PX}_{2}\right)_{j}\left(\mathrm{Q}_{2} \mathrm{R}_{2} \mathrm{X}_{3}\right)_{k}$} \\
\hline$N_{12}$ & $N_{14}$ & $N_{15}$ & $N_{16}$ & $i\left(\mathrm{R}_{3} \mathrm{X}\right)$ & $j\left(\mathrm{PX}_{2}\right)$ & $k\left(\mathrm{Q}_{2} \mathrm{R}_{2} \mathrm{X}_{3}\right)$ & $N_{12}$ & $N_{14}$ & $N_{15}$ & $N_{16}$ & $i\left(\mathrm{R}_{3} \mathrm{X}\right)$ & $j\left(\mathrm{PX}_{2}\right)$ & $k\left(\mathrm{Q}_{2} \mathrm{R}_{2} \mathrm{X}_{3}\right)$ \\
\hline 3 & 2 & 2 & 0 & 0 & $2 \times 0$ & 1 & 2 & 0 & 0 & 1 & 0 & 1 & $\mathbf{0}$ \\
\hline 7 & 2 & 2 & 2 & 0 & $2 \times 1$ & 1 & $?$ & $?$ & $?$ & $?$ & $?$ & $?$ & 1 \\
\hline 7 & 2 & 2 & 2 & 0 & $2 \times 1$ & 1 & 6 & 5 & 2 & 1 & 1 & 1 & 1 \\
\hline 11 & 2 & 2 & 4 & 0 & $2 \times 2$ & 1 & 25 & 19 & 4 & 7 & 5 & 7 & 2 \\
\hline 15 & 2 & 2 & 6 & 0 & $2 \times 3$ & 1 & 27 & 12 & 6 & 8 & 2 & 8 & 3 \\
\hline 23 & 2 & 2 & 10 & 0 & $2 \times 5$ & 1 & 37 & 40 & 10 & 6 & 10 & 6 & 5 \\
\hline 35 & 2 & 2 & 16 & 0 & $2 \times 8$ & 1 & & & & & & & \\
\hline
\end{tabular}
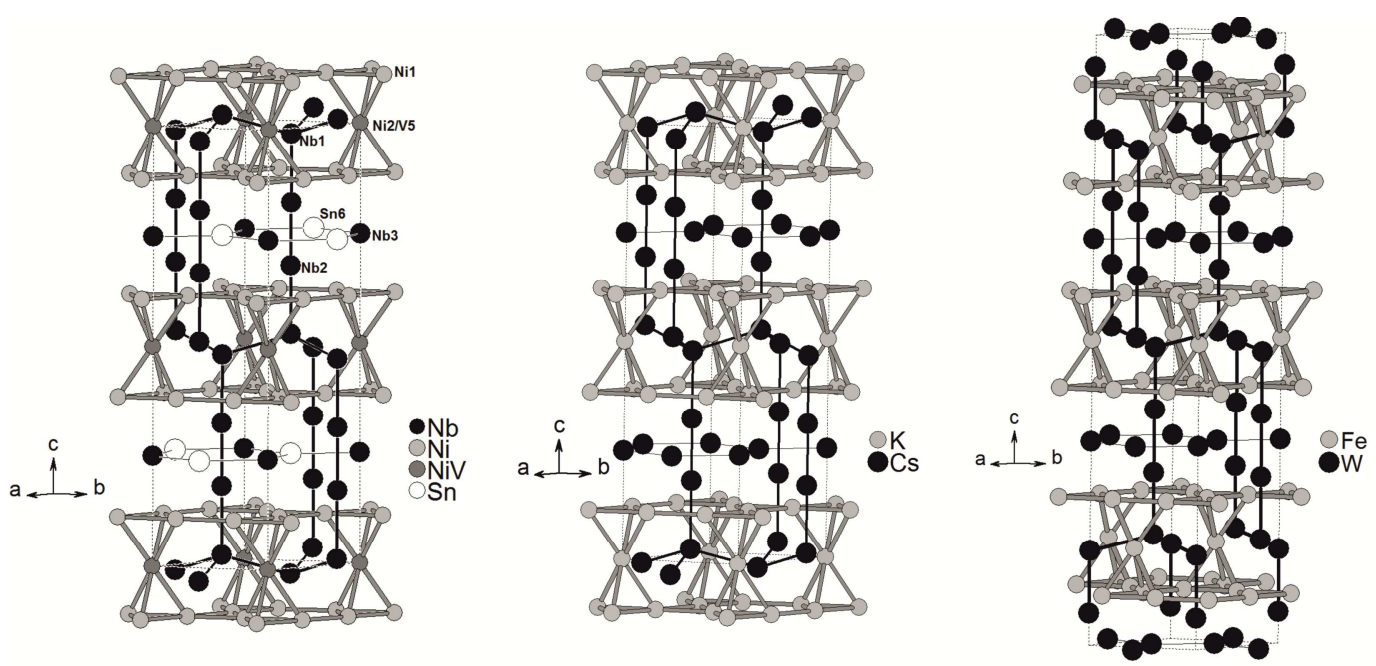

Fig. $3 \mathrm{Nb}_{5} \mathrm{Ni}_{6.84} \mathrm{~V}_{0.16} \mathrm{Sn}_{0.84}$ substructure $\left(h P 26, P 6_{3} / m m c\right)$ of the new stannide, compared with the structures of $\mathrm{Cs}_{6} \mathrm{~K}_{7}\left(h P 26, P 6_{3} / m m c\right)$ and $\mathrm{W}_{6} \mathrm{Fe}_{7}(h R 39, R-3 m)$. Similar structural features are emphasized.

The $\mathrm{Nb}_{5} \mathrm{Ni}_{6.84} \mathrm{~V}_{0.16} \mathrm{Sn}_{0.84} \quad$ substructure ( $h P 26$, $\left.P 6_{3} / m m c\right)$ of the new stannide can be written as $\left(\mathrm{P}_{2} \mathrm{R}_{2} \mathrm{Q}\right) \mathrm{X}_{7} \mathrm{Q}=\left(\mathrm{PX}_{2}\right)_{2}\left(\mathrm{Q}_{2} \mathrm{R}_{2} \mathrm{X}_{3}\right)_{1}$ and, consequently, it belongs to the family of tetrahedrally close-packed structures. It is a ternary ordering variant of the $\mathrm{Cs}_{6} \mathrm{~K}_{7}$ type [13] and four more structure types with the same type of coordination are known: $\mathrm{Cs}_{6} \mathrm{~K}_{7}(h P 26$, $\left.P 6_{3} / m m c\right), \quad \mathrm{W}_{6} \mathrm{Fe}_{7} \quad(h R 39, \quad R-3 m) \quad(\mu$ phase), $\mathrm{W}_{6}(\mathrm{Fe}, \mathrm{Si}, \mathrm{W})_{7} \quad(o P 26, \quad$ Pbam $) \quad(\mathrm{p}-\sigma$ phase $), \mathrm{Th}_{6} \mathrm{Cd}_{7}$ (oP26, Pbam), and $\mathrm{Nb}_{6}(\mathrm{Ni}, \mathrm{Al}, \mathrm{Nb})_{7}(o P 52$, Pnma) $(\mathrm{M}$ phase). Drawings of these structures are presented in Figs. 3 and 4, where alike structure details are marked in a similar way. Fig. 5 shows the decomposition of the $\mathrm{Nb}_{5} \mathrm{Ni}_{6.84} \mathrm{~V}_{0.16} \mathrm{Sn}_{0.84}$ substructure into fragments characteristic of the prototypes $\mathrm{MgZn}_{2}\left(\mathrm{PX}_{2}, h P 12\right.$, $\left.P 6_{3} / m m c\right)$ and $\mathrm{Zr}_{4} \mathrm{Al}_{3}\left(\mathrm{Q}_{2} \mathrm{R}_{2} \mathrm{X}_{3}, h P 7, P 6 / m m m\right)$.

The $\mathrm{V}_{12} \mathrm{Sn}$ substructure $\left(h P 26, P 6_{3} / m m c\right)$ can be considered as built up by polyhedra with 20 (V5) and
15 (Sn) vertices, which share atoms to form a 3D-framework. A similar description applies to the structure type $\mathrm{Be}_{12} \mathrm{Ti}(\mathrm{hP13}, \mathrm{P6} / \mathrm{mmm})$, for which only one binary representative is known [14], that is built up by 15 -vertex (Be) and 20-vertex (Ti) polyhedra. The two structures are compared on Fig. 6.

\section{Conclusions}

The structure a new multicomponent stannide $\mathrm{Nb}_{4.4} \mathrm{~V}_{1.6} \mathrm{Ni}_{6} \mathrm{Sn}_{0.9}$ was refined from $\mathrm{X}$-ray singlecrystal diffraction data. It was found to be formed by two substructures: $\mathrm{Nb}_{5} \mathrm{Ni}_{6.84} \mathrm{~V}_{0.16} \mathrm{Sn}_{0.84}$ and $\mathrm{V}_{12} \mathrm{Sn}$, in the approximate ratio $9: 1$. The substructure $\mathrm{Nb}_{5} \mathrm{Ni}_{6.84} \mathrm{~V}_{0.16} \mathrm{Sn}_{0.84}$ is a ternary ordering variant of the $\mathrm{Cs}_{6} \mathrm{~K}_{7}$ type and belongs to the family of tetrahedrally close-packed structures, also known as Frank-Kasper 

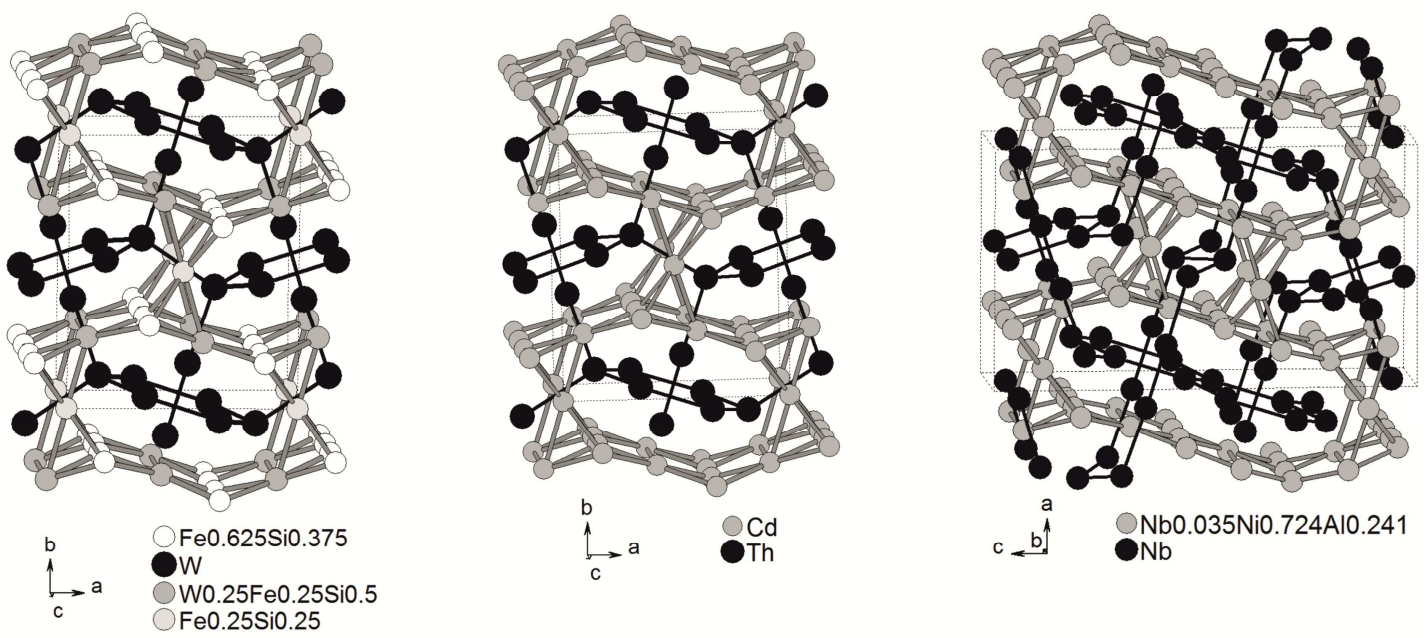

Fig. 4 Crystal structures of $\mathrm{W}_{6}(\mathrm{Fe}, \mathrm{Si}, \mathrm{W})_{7}(o P 26, P b a m), \mathrm{Th}_{6} \mathrm{Cd}_{7},(o P 26, P b a m)$ and $\mathrm{Nb}_{6}(\mathrm{Ni}, \mathrm{Al}, \mathrm{Nb})_{7}(o P 52$, Pnma). Similar structural features are emphasized.

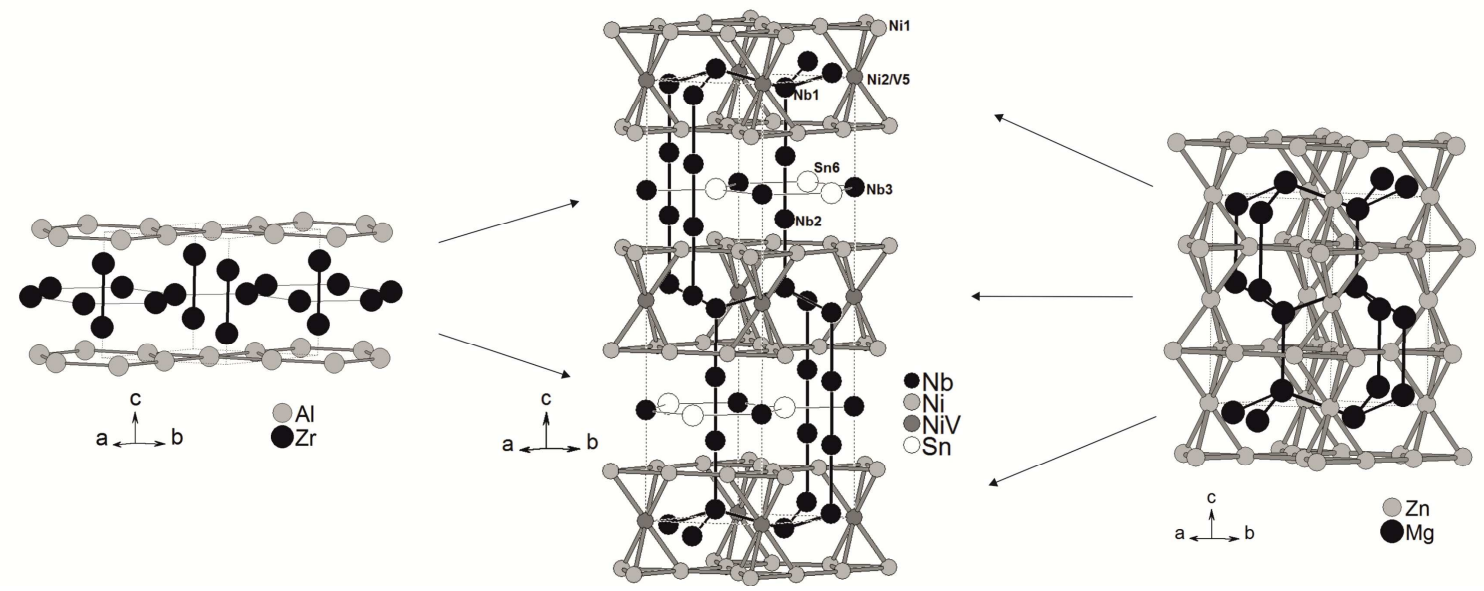

Fig. 5 The t.c.p. substructure $\mathrm{Nb}_{5} \mathrm{Ni}_{6.84} \mathrm{~V}_{0.16} \mathrm{Sn}_{0.84}\left(h P 26, P 6_{3} / m m c\right)$ presented as a combination of $\mathrm{MgZn}_{2}$ $\left(h P 12, P 6_{3} / m m c\right)$ and $\mathrm{Zr}_{4} \mathrm{Al}_{3^{-}}(h P 7, P 6 / \mathrm{mmm})$ type fragments.
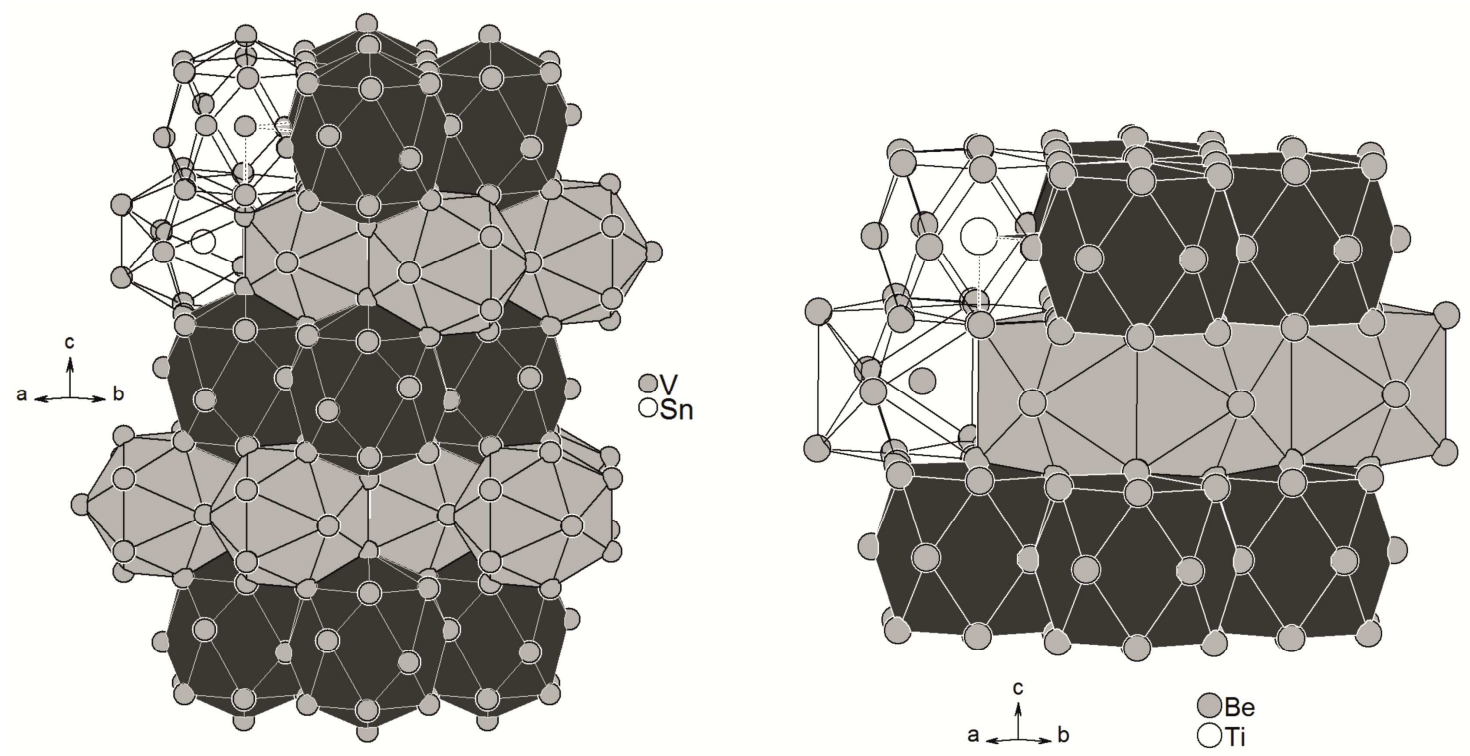

Fig. $6 \mathrm{~V}_{12} \mathrm{Sn}$ substructure $\left(h P 26, P 6_{3} / m m c\right)$ of the new stannide and structure of $\mathrm{Be}_{12} \mathrm{Ti}(h P 13, P 6 / m m m)$. 
phases. The $\mathrm{Nb}$ atoms center 14-, 15-, and 16-vertex Frank-Kasper polyhedra, the $\mathrm{Sn}$ atoms 15-vertex Frank-Kasper polyhedra, and the $\mathrm{Ni}$ or $\mathrm{V}$ atoms icosahedra (12-vertes Frank-Kasper polyhedra). In the minority substructure $\mathrm{V}_{12} \mathrm{Sn}$, the $\mathrm{V}$ atoms are surrounded by 12-, 14-, and 15-vertex Frank-Kasper polyhedra and by 20 -vertex polyhedra, and the $\mathrm{Sn}$ atoms by 15 -vertex Frank-Kasper polyhedra. Similar polyhedra are observed for $\mathrm{Be}_{12} \mathrm{Ti}(\mathrm{hP} 13, \mathrm{P} 6 / \mathrm{mmm})$.

One of the crystal chemical features of structures built from Frank-Kasper polyhedra is the linear dependence of the weight-averaged coordination number on the icosahedron content, known as the Yarmolyuk-Kripyakevich criterion. The atoms in these structures are surrounded by P- (16 vertices), Q- (15 vertices), R- (14 vertices), and X-type (12 vertices) Frank-Kasper polyhedra. The structural formula of any of the compounds can be written as $\left(\mathrm{R}_{3} \mathrm{X}\right)_{i}\left(\mathrm{PX}_{2}\right)_{j}\left(\mathrm{Q}_{2} \mathrm{R}_{2} \mathrm{X}_{3}\right)_{k}$, which represents a combination of the prototypes $\mathrm{Cr}_{3} \mathrm{Si}(c P 8, P m-3 n)$, $\mathrm{MgZn}_{2}\left(h P 12, P 6_{3} / m m c\right)$, and $\mathrm{Zr}_{4} \mathrm{Al}_{3}$ (hP7, P6/mmm).

Considering the Yarmolyuk-Kripyakevich criterion, rewritten as $\mathrm{P}_{j} \mathrm{Q}_{2 k} \mathrm{R}_{3 i+2 k} \mathrm{X}_{i+2 j+3 k}$, solutions of the equation that relates the numbers of different polyhedra $\left(N_{12}=2 \cdot N_{16}+7 / 6 \cdot N_{15}+1 / 3 \cdot N_{14}\right)$ were derived, limited to values of $N_{16}, N_{15}, N_{14}, N_{12} \leq 50$. It was found that, among the different possible combinations obeying the Yarmolyuk-Kripyakevich rule, only one combination has been experimentally observed for a particular number of icosahedra. In most cases the simplest combination, with minimal average coordination number and lowest number of fragments (i.e. $i+j+k$ ) is preferred. In the general case, such simple solutions can be found using the following formulas: $\mathrm{P}_{1+2 n} \mathrm{Q}_{2} \mathrm{R}_{2} \mathrm{X}_{5+4 n}, \mathrm{P}_{1+2 n} \mathrm{Q}_{2} \mathrm{R}_{5} \mathrm{X}_{6+4 n}$, $\mathrm{P}_{1+2 n+1} \mathrm{Q}_{2} \mathrm{R}_{2} \mathrm{X}_{7+4 n}$, and $\mathrm{P}_{1+2 n} \mathrm{Q}_{4} \mathrm{R}_{4} \mathrm{X}_{8+4 n}$.

In addition to the observation made by Hellner and Pearson that $\mathrm{Q}_{2} \mathrm{R}_{2} \mathrm{X}_{3}\left(\mathrm{Zr}_{4} \mathrm{Al}_{3}\right)$ fragments are needed to stabilize the structures, new features were found. Distributing all known combinations $\left(\mathrm{R}_{3} \mathrm{X}\right)_{i}\left(\mathrm{PX}_{2}\right)_{j}\left(\mathrm{Q}_{2} \mathrm{R}_{2} \mathrm{X}_{3}\right)_{k}$ into groups according to the number of different fragments, some regularities were observed. The values of $i, j$, and $k$ revealed series, where every number is the sum of the two preceding ones: $0,1,1,2,3,5,8, \ldots$, known as the Fibonacci sequence.

\section{Acknowledgments}

This work was supported by the Ministry of Education and Sciences of Ukraine under the grants
No. 0112 U001279 and No. 0112U001280, and by a Leonhard-Euler-Stipendien program (V.G.) at the Technical University of Munich.

\section{References}

[1] P. Villars, K. Cenzual (Eds.), Pearson's Crystal Data: Crystal Structure Database for Inorganic Compounds, ASM International, Materials Park, Ohio, USA, Release 2012/13.

[2] F.C. Frank, J.S. Kasper, Acta Crystallogr. 11 (1958) 184-190.

[3] F.C. Frank, J.S. Kasper, Acta Crystallogr. 12 (1959) 483-499.

[4] Ya.P. Yarmolyuk, P.I. Kripyakevich, Sov. Phys. Crystallogr. 19 (1974) 334-337.

[5] D.P. Shoemaker, C. Brink Shoemaker, Acta Crystallogr. B. 42 (1986) 3-11.

[6] E. Parthé, L. Gelato, B. Chabot, M. Penzo, K. Cenzual, R. Gladyshevskii, TYPIX: Standardized Data and Crystal Chemical Characterization of Inorganic Structure Types, Springer-Verlag, Heidelberg, Germany, 1993, 1994, Vol. 1-4, 1596 p.

[7] M.A.S. Boff, G.L.F. Fraga, D.E. Brandao, A.A. Gomes, J. Magn. Magn. Mater. 153 (1996) 135-140.

[8] Y. Fujita, K. Endo, M. Terada, M. Kimura, J. Phys. Chem. Solids 33 (1972) 1443-1446.

[9] V. Gvozdetskyi, R. Gladyshevskii, V. Hlukhyy, T. Fässler, to be published.

[10] V.N. Eremenko, S.B. Prima, L.A. Tret'yachenko, P.A. Verkhovodov, Sov. Powder Metall. Met. Ceram. 25 (1986) 977-981.

[11] CrysAlis RED, Scale3 / ABSPACK. Version 1.171.33.34d. Oxford Diffraction Poland Sp. $z$ o.o., 2009.

[12] G.M. Sheldrick, Acta Crystallogr. A. 64 (2008) 112-122.

[13] A. Simon, W. Brämer, B. Hillenkötter, H.J. Kullmann, Z. Anorg. Allg. Chem. 419 (1976) 253-274.

[14] R.F. Raeuchle, R.E. Rundle, Acta Crystallogr. 5 (1952) 85-93.

[15] D.X. Li, K.H. Kuo, Acta Crystallogr. B 42 (1986) 152-159.

[16] M.D. Sikirić, O. Delgado-Friedrichs, M. Deza, Acta Crystallogr. A 66 (2010) 602-615.

[17] E. Hellner, W.B. Pearson, J. Solid State Chem. 70 (1987) 241-248. 